\title{
Modified hybrid iterative methods for generalized mixed equilibrium, variational inequality and fixed point problems
}

\author{
Jong Soo Jung \\ Department of Mathematics, Dong-A University, Busan 49315, Korea.
}

Communicated by Y. J. Cho

\begin{abstract}
In this paper, we introduce two modified hybrid iterative methods (one implicit method and one explicit method) for finding a common element of the set of solutions of a generalized mixed equilibrium problem, the set of solutions of a variational inequality problem for a continuous monotone mapping and the set of fixed points of a continuous pseudocontractive mapping in Hilbert spaces, and show under suitable control conditions that the sequences generated by the proposed iterative methods converge strongly to a common element of three sets, which solves a certain variational inequality. As a direct consequence, we obtain the unique minimum-norm common point of three sets. The results in this paper substantially improve upon, develop and complement the previous well-known results in this area. (C)2017 All rights reserved.
\end{abstract}

Keywords: Hybrid iterative method, generalized mixed equilibrium problem, continuous monotone mapping, continuous pseudocontractive mapping, variational inequality, fixed point, $\rho$-Lipschitzian and $\eta$-strongly monotone mapping, metric projection.

2010 MSC: 49J30, 49J40, 47H09, 47H10, 47J20, 47J25, 47J05, 49M05.

\section{Introduction}

Let $\mathrm{H}$ be a real Hilbert space with inner product $\langle\cdot, \cdot\rangle$ and induced norm $\|\cdot\|$. Let $\mathrm{C}$ be a nonempty closed convex subset of $\mathrm{H}$ and let $\mathrm{T}: \mathrm{C} \rightarrow \mathrm{C}$ be self-mapping on $\mathrm{C}$. We denote by Fix $(\mathrm{T})$ the set of fixed points of $\mathrm{T}$.

Let $\mathrm{B}: \mathrm{C} \rightarrow \mathrm{H}$ be a nonlinear mapping, let $\varphi: \mathrm{C} \rightarrow \mathbb{R}$ be a function and let $\Theta$ be a bifunction of $\mathrm{C} \times \mathrm{C}$ into $\mathbb{R}$, where $\mathbb{R}$ is the set of real numbers.

Then, we consider the following generalized mixed equilibrium problem (for short, GMEP) of finding $x \in \mathrm{C}$ such that

$$
\Theta(x, y)+\langle B x, y-x\rangle+\varphi(y)-\varphi(x) \geqslant 0, \quad \forall y \in C,
$$

which was introduced by Peng and Yao [22] (also see [18, 42]). The set of solutions of the problem (1.1) is denoted by $\operatorname{GMEP}(\Theta, \varphi, \mathrm{B})$. Here some special cases of the problem (1.1) are stated as follows:

If $\varphi=0$, then the problem (1.1) reduces the following generalized equilibrium problem (for short, GEP) of finding $x \in C$ such that

$$
\Theta(x, y)+\langle B x, y-x\rangle \geqslant 0, \quad \forall y \in C,
$$

which was studied by Takahashi and Takahashi [29]. The set of solutions of the problem (1.2) is denoted

Email address: jungjs@dau.ac.kr (Jong Soo Jung)

doi:10.22436/jnsa.010.07.30 
by $\operatorname{GEP}(\Theta, B)$.

If $B=0$, then the problem (1.1) reduces the following mixed equilibrium problem (for short, $\mathrm{MEP}$ ) of finding $x \in C$ such that

$$
\Theta(x, y)+\varphi(y)-\varphi(x) \geqslant 0, \quad \forall y \in C,
$$

which was studied by Ceng and Yao [5] (see also [39]). The set of solutions of the problem (1.3) is denoted by $\operatorname{MEP}(\Theta, \varphi)$.

If $\varphi=0$ and $\mathrm{B}=0$, then the problem (1.1) reduces the following equilibrium problem (for short, EP) of finding $x \in C$ such that

$$
\Theta(x, y) \geqslant 0, \quad \forall y \in C .
$$

The set of solutions of the problem (1.4) is denoted by $\operatorname{EP}(\Theta)$.

If $\varphi=0$ and $\Theta(x, y)=0$ for all $x, y \in C$, the problem (1.1) reduces the following variational inequality problem (for short, VIP) of finding $x \in C$ such that

$$
\langle\mathrm{B} x, \mathrm{y}-\mathrm{x}\rangle \geqslant 0, \quad \forall \mathrm{y} \in \mathrm{C} .
$$

The set of solutions of the problem (1.5) is denoted by $\mathrm{VI}(\mathrm{C}, \mathrm{B})$.

The GMEP (1.1) is very general in the sense that it includes, as special cases, fixed point problems, optimization problems, variational inequality problems, minmax problems, Nash equilibrium problems in noncooperative games and others, see for example $[2,5,9,10]$.

As we all know, the convex feasibility problem (CFP) is the problem of finding a point in the (nonempty) intersection $C=\cap_{i=1}^{m} C_{i}$ of a finite number of closed convex sets $C_{i}(i=1, \cdots, m)$. The split common fixed point problem (SCFP) is a generalization of the split feasibility problem (SFP) and the problem (CFP). Several iterative methods for solving the problem (SCFP) for nonlinear mappings were developed; see for example $[7,31,33,40]$ and the references therein.

Recently, many authors considered iterative methods for finding a common point of solution sets of the problems GMEP (1.1), GEP (1.2), MEP (1.3), EP (1.4) and VIP (1.5) and fixed point sets of nonlinear mappings as special cases of the problem (CFP). In particular, in order to study the EP (1.4) coupled with the fixed point problem, many authors have introduced iterative methods for finding a common element of the set of the solutions of the EP (1.4) and the set of fixed points of a countable family of nonexpansive mappings; see $[6,8,13,23,24,27,28,32,37,38]$ and the references therein.

In 2008, Su et al. [25] gave an iterative method for the EP (1.4), the VIP (1.5) for an inverse-strongly monotone mapping $F$ and nonexpansive mapping $S$ and proved strong convergence to a point $z$ in $\operatorname{EP}(\Theta) \cap$ $\mathrm{VI}(\mathrm{C}, \mathrm{F}) \cap \mathrm{Fix}(\mathrm{S})$. In 2009, Yao et al. [36] considered an iterative method for the MEP (1.3), the VIP (1.5) for a Lipschitz and relaxed-cocoercive mapping $F$ and a sequence $\left\{S_{n}\right\}$ of nonexpansive mappings, and proved strong convergence to a point $z \in \cap_{n=1}^{\infty} \operatorname{Fix}\left(S_{n}\right) \cap \operatorname{MEP}(\Theta, \varphi) \cap \operatorname{VI}(C, F)$. In 2008, Peng and Yao [22] studied an iterative method for the GMEP (1.1) related to an $\alpha$-inverse-strongly monotone mapping B, the VIP (1.5) for a monotone and Lipschitz continuous mapping $F$ and a nonexpansive mapping $S$, and proved strong convergence to a point $z \in \operatorname{GMEP}(\Theta, \varphi, \mathrm{B}) \cap \operatorname{VI}(\mathrm{C}, \mathrm{F}) \cap \operatorname{Fix}(\mathrm{S})$. In 2010, by using the method of Yao et al. [39], Jaiboon and Kumam [12] also introduced an iterative method related to optimization problem for the MEP (1.3), the VIP (1.5) for an $\alpha$-inverse-strongly monotone mapping F and a sequence $\left\{S_{n}\right\}$ of nonexpansive mappings, and showed strong convergence to a point $z \in \cap_{n=1}^{\infty}$ Fix $\left(S_{n}\right) \cap$ $\operatorname{MEP}(\Theta, \varphi) \cap \operatorname{VI}(\mathrm{C}, \mathrm{F})$.

In 2007, Tada and Takahashi [27] considered an iterative method for the EP (1.4) and nonexpansive mapping $S$ and proved weak convergence to a point $w \in E P(\Theta) \cap \operatorname{Fix}(S)$. In 2008, Moudafi [21] proposed an iterative method for the GEP (1.2) related to an $\alpha$-inverse-strongly monotone mapping B and nonexpansive mapping $S$ and showed weak convergence to a point $w \in \operatorname{GEP}(\Theta, B) \cap$ Fix $(S)$. In 2009, Ceng et al. [3] provided an iterative method for the EP (1.4) and k-strictly pseudocontractive mapping $T$ and proved weak convergence to a point $w \in \mathrm{EP}(\Theta) \cap \mathrm{Fix}(\mathrm{T})$. In 2015, Lv [19] also studied an iterative method for the GEP (1.2) and k-strictly pseudocontractive mapping $T$ and proved weak convergence to a point $w \in \operatorname{GEP}(\Theta) \cap \operatorname{Fix}(\mathrm{T})$. 
In 2003, Takahashi and Toyoda [30] introduced an iterative method for the VIP (1.4) related to an $\alpha$ inverse-strongly monotone mapping $F$ and nonexpansive mapping $S$ and established weak convergence to a point $w \in \operatorname{Fix}(S) \cap V I(C, F)$.

In 2012, Jung [16] considered an iterative method for GMEP (1.1) related to a $\beta$-inverse-strongly monotone mapping B, the VIP (1.5) for an $\alpha$-inverse-strongly monotone mapping and k-strictly pseudocontractive mapping $T$ and proved weak convergence to a point $w \in \operatorname{GMEP}(\Theta, \varphi, B) \cap \operatorname{VI}(\mathrm{C}, \mathrm{F}) \cap$ Fix $(\mathrm{T})$. In 2015 Jung [17] also proposed an iterative method for GMEP (1.1) related to a continuous monotone mapping $\mathrm{B}$, the VIP (1.5) for a continuous monotone mapping $\mathrm{F}$ and a continuous pseudocontractive mapping $\mathrm{T}$ and proved weak convergence to a point $w \in \operatorname{GMEP}(\Theta, \varphi, \mathrm{B}) \cap \mathrm{VI}(\mathrm{C}, \mathrm{F}) \cap \mathrm{Fix}(\mathrm{T})$.

In 2012, by using Yamada's hybrid steepest-descent method [35] and Jung's viscosity iterative method [14], Jung [15] introduced new implicit and explicit iterative methods for finding a common element of the set of solutions of the MEP (1.3) and the set of fixed points of a k-strictly pseudocontractive mapping T and proved strong convergence to a point $z \in \operatorname{MEP}(\Theta, \varphi) \cap \mathrm{Fix}(\mathrm{T})$. In particular, in 2012, by combining Colao et al.'s hybrid viscosity iterative method [8] and Yamada's hybrid steepest-descent method [35], Ceng et al. [4] proposed a hybrid iterative method for finding a common element of the set of solutions of the GMEP (1.1) related to an $\alpha$-inverse-strongly monotone mapping $B$ and the set of fixed points of a finite family of nonexpansive mappings $\left\{T_{i}\right\}_{i=1}^{N}$ and showed strong convergence to a point $z \in \cap_{i=1}^{N} \operatorname{Fix}\left(T_{i}\right) \cap$ $\operatorname{GMEP}(\Theta, \varphi, \mathrm{B})$ which is a unique solution of certain variational inequality related to Lipschitzian and strongly monotone mapping $\mathrm{G}$.

In this paper, inspired and motivated by above-mentioned results, we introduce two new modified hybrid iterative methods (one implicit method and one explicit method) for finding a common element of the solution set $\operatorname{GMEP}(\Theta, \varphi, \mathrm{B})$ of the GMEP (1.1) related to a continuous monotone mapping $\mathrm{B}$, the solution set $\mathrm{VI}(\mathrm{C}, \mathrm{F})$ of the VIF (1.5) for a continuous monotone mapping $\mathrm{F}$ and the fixed point set Fix $(\mathrm{T})$ of a continuous pseudocontractive mapping $\mathrm{T}$ in a Hilbert space. We show that under suitable conditions, the sequences generated by the proposed iterative methods converge strongly to a common element of $\Omega:=\operatorname{GMEP}(\Theta, \varphi, \mathrm{B}) \cap \mathrm{VI}(\mathrm{C}, \mathrm{F}) \cap \operatorname{Fix}(\mathrm{T})$, which is a solution of a certain variational inequality. As a direct consequence, we find the unique solution of the minimization norm problem

$$
\left\|x^{*}\right\|=\min \{\|x\|: x \in \Omega\} .
$$

The results in this paper develop, improve upon and complement of the recent results announced by several authors in this direction.

\section{Preliminaries and lemmas}

Let $\mathrm{H}$ be a real Hilbert space and let $\mathrm{C}$ be a nonempty closed convex subset of $\mathrm{H}$. In the following, we write $x_{n} \rightarrow x$ to indicate that the sequence $\left\{x_{n}\right\}$ converges weakly to $x . x_{n} \rightarrow x$ implies that $\left\{x_{n}\right\}$ converges strongly to $x$.

We recall $([1,11])$ that a mapping $F$ of $\mathrm{C}$ into $\mathrm{H}$ is called

(i) Lipschitzian, if there exists a constant $k \geqslant 0$ such that

$$
\|F x-F y\| \leqslant \kappa\|x-y\| \quad \forall x, y \in C ;
$$

(ii) monotone, if $\langle x-y, F x-F y\rangle \geqslant 0, \quad \forall x, y \in C$;

(iii) $\alpha$-inverse-strongly monotone, if there exists a constant $\alpha>0$ such that

$$
\langle x-y, F x-F y\rangle \geqslant \alpha\|F x-F y\|^{2}, \quad \forall x, y \in C ;
$$

(iv) $\eta$-strongly monotone, if there exists a positive real number $\eta$ such that

$$
\langle x-y, F x-F y\rangle \geqslant \eta\|x-y\|^{2}, \quad \forall x, y \in C .
$$


We note that if $F$ is an $\alpha$-inverse-strongly monotone mapping of $\mathrm{C}$ into $\mathrm{H}$, then it is obvious that $\mathrm{F}$ is $\frac{1}{\alpha}$-Lipschitz continuous, that is, $\|F x-F y\| \leqslant \frac{1}{\alpha}\|x-y\|$ for all $x, y \in C$. Clearly, the class of monotone mappings includes the class of $\alpha$-inverse-strongly monotone mappings.

We recall ([1]) that a mapping $\mathrm{T}: \mathrm{C} \rightarrow \mathrm{H}$ is said to be pseudocontractive, if

$$
\|T x-T y\|^{2} \leqslant\|x-y\|^{2}+\|(I-T) x-(I-T) y\|^{2}, \quad \forall x, y \in C,
$$

and $T$ is said to be $k$-strictly pseudocontractive, if there exists a constant $k \in[0,1)$ such that

$$
\|\mathrm{T} x-\mathrm{T} y\|^{2} \leqslant\|x-y\|^{2}+k\|(I-T) x-(I-T) y\|^{2}, \quad \forall x, y \in C,
$$

where I is the identity mapping. The class of k-strictly pseudocontractive mappings includes the class of nonexpansive mappings as a subclass. That is, $T$ is nonexpansive, (i.e., $\|T x-T y\| \leqslant\|x-y\|, \forall x, y \in C$ ) if and only if $\mathrm{T}$ is 0 -strictly pseudocontractive.

For solving the GMEP (1.1), the MEP (1.2), and the EP (1.3) for a bifunction $\Theta: C \times C \rightarrow \mathbb{R}$, let us assume that $\Theta$ satisfies the following conditions:

(A1) $\Theta(x, x)=0$ for all $x \in C$;

(A2) $\Theta$ is monotone, that is, $\Theta(x, y)+\Theta(y, x) \leqslant 0$ for all $x, y \in C$;

(A3) for each $x, y, z \in C$,

$$
\limsup _{t \downarrow 0} \Theta(t z+(1-t) x, y) \leqslant \Theta(x, y) ;
$$

(A4) for each $x \in C, y \mapsto \Theta(x, y)$ is convex and lower semicontinuous.

We can prove the following lemma by using the same method as in [18,42], and so we omit its proof.

Lemma 2.1. Let $\mathrm{C}$ be a nonempty closed convex subset of $\mathrm{H}$. Let $\Theta$ be a bifunction form $\mathrm{C} \times \mathrm{C}$ to $\mathbb{R}$ satisfies (A1)-(A4) and $\varphi: \mathrm{C} \rightarrow \mathbb{R}$ be a proper lower semicontinuous and convex function. Let $\mathrm{B}: \mathrm{C} \rightarrow \mathrm{H}$ be a continuous monotone mapping. Then, for $\mathrm{r}>0$ and $\mathrm{x} \in \mathrm{H}$, there exists $\mathrm{u} \in \mathrm{C}$ such that

$$
\Theta(u, y)+\langle B u, y-u\rangle+\varphi(y)-\varphi(u)+\frac{1}{r}\langle y-u, u-x\rangle \geqslant 0, \forall y \in C .
$$

Define a mapping $\mathrm{K}_{\mathrm{r}}: \mathrm{H} \rightarrow \mathrm{C}$ as follows:

$$
\mathrm{K}_{\mathrm{r}} \mathrm{x}=\left\{\mathrm{u} \in \mathrm{C}: \Theta(\mathrm{u}, \mathrm{y})+\langle\mathrm{Bu}, \mathrm{y}-\mathrm{u}\rangle+\varphi(\mathrm{y})-\varphi(\mathrm{u})+\frac{1}{\mathrm{r}}\langle\mathrm{y}-\mathrm{u}, \mathrm{u}-\mathrm{x}\rangle \geqslant 0, \forall \mathrm{y} \in \mathrm{C}\right\}
$$

for all $\mathrm{x} \in \mathrm{H}$ and $\mathrm{r}>0$. Then, the following hold:

(1) For each $x \in H, K_{r} x \neq \emptyset$;

(2) $\mathrm{K}_{\mathrm{r}}$ is single-valued;

(3) $\mathrm{K}_{\mathrm{r}}$ is firmly nonexpansive, that is, for any $\mathrm{x}, \mathrm{y} \in \mathrm{H}$,

(4) $\mathrm{F}\left(\mathrm{K}_{\mathrm{r}}\right)=\operatorname{GMEP}(\Theta, \varphi, \mathrm{B})$;

$$
\left\|K_{r} x-K_{r} y\right\|^{2} \leqslant\left\langle K_{r} x-K_{r} y, x-y\right\rangle ;
$$

(5) $\operatorname{GMEP}(\Theta, \varphi, \mathrm{B})$ is closed and convex.

We need the following lemmas for the proof of our main results.

Lemma 2.2 ([41]). Let $\mathrm{C}$ be a closed convex subset of a real Hilbert space $\mathrm{H}$. Let $\mathrm{F}: \mathrm{C} \rightarrow \mathrm{H}$ be a continuous monotone mapping. Then, for $\mathrm{r}>0$ and $\mathrm{x} \in \mathrm{H}$, there exists $z \in \mathrm{C}$ such that

$$
\langle\mathrm{F} z, \mathrm{y}-z\rangle+\frac{1}{\mathrm{r}}\langle\mathrm{y}-z, z-\mathrm{x}\rangle \geqslant 0, \quad \forall \mathrm{y} \in \mathrm{C} .
$$

For $\mathrm{r}>0$ and $\mathrm{x} \in \mathrm{H}$, define $\mathrm{F}_{\mathrm{r}}: \mathrm{H} \rightarrow \mathrm{C}$ by

$$
\mathrm{F}_{\mathrm{r}} x=\left\{z \in \mathrm{C}:\langle\mathrm{F} z, y-z\rangle+\frac{1}{\mathrm{r}}\langle\mathrm{y}-z, z-x\rangle \geqslant 0, \quad \forall \mathrm{y} \in \mathrm{C}\right\} .
$$

Then the following hold: 
(i) $\mathrm{F}_{\mathrm{r}}$ is single-valued;

(ii) $\mathrm{F}_{\mathrm{r}}$ is firmly nonexpansive, that is,

$$
\left\|F_{r} x-F_{r} y\right\|^{2} \leqslant\left\langle F_{r} x-F_{r} y, x-y\right\rangle, \quad \forall x, y \in H ;
$$

(iii) $\operatorname{Fix}\left(\mathrm{F}_{\mathrm{r}}\right)=\mathrm{VI}(\mathrm{C}, \mathrm{F})$;

(iv) $\mathrm{V}(\mathrm{I}, \mathrm{F})$ is a closed convex subset of $\mathrm{C}$.

Lemma 2.3 ([41]). Let $\mathrm{C}$ be a closed convex subset of a real Hilbert space $\mathrm{H}$. Let $\mathrm{T}: \mathrm{C} \rightarrow \mathrm{H}$ be a continuous pseudocontractive mapping. Then, for $\mathrm{r}>0$ and $\mathrm{x} \in \mathrm{H}$, there exists $z \in \mathrm{C}$ such that

$$
\langle\mathrm{T} z, \mathrm{y}-z\rangle-\frac{1}{\mathrm{r}}\langle\mathrm{y}-z,(1+\mathrm{r}) z-\mathrm{x}\rangle \leqslant 0, \quad \forall \mathrm{y} \in \mathrm{C} .
$$

For $\mathrm{r}>0$ and $\mathrm{x} \in \mathrm{H}$, define $\mathrm{T}_{\mathrm{r}}: \mathrm{H} \rightarrow \mathrm{C}$ by

$$
\mathrm{T}_{\mathrm{r}} x=\left\{z \in \mathrm{C}:\langle\mathrm{T} z, \mathrm{y}-z\rangle-\frac{1}{\mathrm{r}}\langle\mathrm{y}-z,(1+\mathrm{r}) z-x\rangle \leqslant 0, \quad \forall \mathrm{y} \in \mathrm{C}\right\} .
$$

Then the following hold:

(i) $\mathrm{T}_{\mathrm{r}}$ is single-valued;

(ii) $\mathrm{T}_{\mathrm{r}}$ is firmly nonexpansive, that is,

$$
\left\|T_{r} x-T_{r} y\right\|^{2} \leqslant\left\langle T_{r} x-T_{r} y, x-y\right\rangle, \quad \forall x, y \in H ;
$$

(iii) $\operatorname{Fix}\left(\mathrm{T}_{\mathrm{r}}\right)=\operatorname{Fix}(\mathrm{T})$;

(iv) Fix $(\mathrm{T})$ is a closed convex subset of $\mathrm{C}$.

Lemma 2.4 ([34]). Let $\left\{s_{n}\right\}$ be a sequence of non-negative real numbers satisfying

$$
s_{n+1} \leqslant\left(1-\lambda_{n}\right) s_{n}+\beta_{n}, \quad \forall n \geqslant 1,
$$

where $\left\{\lambda_{n}\right\}$ and $\left\{\beta_{n}\right\}$ satisfy the following conditions:

(i) $\left\{\lambda_{n}\right\} \subset[0,1]$ and $\sum_{n=1}^{\infty} \lambda_{n}=\infty$ or, equivalently, $\prod_{n=1}^{\infty}\left(1-\lambda_{n}\right)=0$;

(ii) $\limsup _{n \rightarrow \infty} \frac{\beta_{n}}{\lambda_{n}} \leqslant 0$ or $\sum_{n=1}^{\infty}\left|\beta_{n}\right|<\infty$.

Then $\lim _{n \rightarrow \infty} s_{n}=0$.

The following lemma is easily proven by property of inner product.

Lemma 2.5. In a Hilbert space, there holds the inequality

$$
\|x+y\|^{2} \leqslant\|x\|^{2}+2\langle y, x+y\rangle, \quad \forall x, y \in H .
$$

Lemma 2.6 ([26]). Let $\left\{x_{n}\right\}$ and $\left\{y_{n}\right\}$ be bounded sequences in a Banach space $E$ and $\left\{\gamma_{n}\right\}$ be a sequence in $[0,1]$ which satisfies the following condition:

$$
0<\liminf _{n \rightarrow \infty} \gamma_{n} \leqslant \limsup _{n \rightarrow \infty} \gamma_{n}<1 .
$$

Suppose that $x_{n+1}=\gamma_{n} x_{n}+\left(1-\gamma_{n}\right) y_{n}$, for all $n \geqslant 1$ and

$$
\limsup _{n \rightarrow \infty}\left(\left\|y_{n+1}-y_{n}\right\|-\left\|x_{n+1}-x_{n}\right\|\right) \leqslant 0 .
$$

Then $\lim _{n \rightarrow \infty}\left\|y_{n}-x_{n}\right\|=0$. 
The following lemma can be easily proven, and therefore, we omit the proof.

Lemma 2.7. Let $\mathrm{V}: \mathrm{C} \rightarrow \mathrm{H}$ be an l-Lipschitzian mapping with constant $\mathrm{l} \geqslant 0$, and $\mathrm{G}: \mathrm{C} \rightarrow \mathrm{H}$ be a $\mathrm{k}$-Lipschitzian and $\eta$-strongly monotone mapping with constants $\mathrm{k}$ and $\eta>0$. Then for $0 \leqslant \gamma \mathrm{l}<\mu \eta$,

$$
\langle(\mu \mathrm{G}-\gamma \mathrm{V}) x-(\mu \mathrm{G}-\gamma \mathrm{V}) \mathrm{y}, x-y\rangle \geqslant(\mu \eta-\gamma l)\|x-y\|^{2}, \quad \forall x, y \in C .
$$

That is, $\mu \mathrm{G}-\gamma \mathrm{V}$ is strongly monotone with constant $\mu \eta-\gamma \mathrm{l}$.

Finally, we need the following lemma (see [35] for the proof).

Lemma 2.8. Let $\mathrm{C}$ be a nonempty closed subspace of a Hilbert space $\mathrm{H}$. Let $\mathrm{G}: \mathrm{C} \rightarrow \mathrm{C}$ be a $\mathrm{k}$-Lipschitzian and $\eta$-strongly monotone mapping with constants $\mathrm{k}>0$ and $\eta>0$. Let $0<\mu<\frac{2 \eta}{\kappa^{2}}$ and $0<\mathrm{t}<\rho \leqslant 1$. Then $\mathrm{S}:=\rho \mathrm{I}-\mathrm{t} \mu \mathrm{G}: \mathrm{C} \rightarrow \mathrm{C}$ is a contraction with contractive constant $\rho-\mathrm{t} \tau$, where $\tau=1-\sqrt{1-\mu\left(2 \eta-\mu \mathrm{K}^{2}\right)}$.

\section{Main results}

Throughout the rest of this paper, we always assume the following:

- $\mathrm{H}$ is a real Hilbert space;

- $\mathrm{C}$ is a nonempty closed subspace of $\mathrm{H}$;

- $\Theta$ is a bifunction from $C \times C \rightarrow \mathbb{R}$ satisfying (A1)-(A4);

- $\mathrm{B}: \mathrm{C} \rightarrow \mathrm{H}$ is a continuous monotone mapping;

- $\mathrm{F}: \mathrm{C} \rightarrow \mathrm{H}$ is a continuous monotone mapping;

- $\mathrm{VI}(\mathrm{C}, \mathrm{F})$ is the set of the variational inequality problem (1.1) for $\mathrm{F}$;

- $\mathrm{T}: \mathrm{C} \rightarrow \mathrm{C}$ is a continuous pseudocontractive mapping with $\operatorname{Fix}(\mathrm{T}) \neq \emptyset$;

- $\mathrm{K}_{\mathrm{r}_{\mathrm{t}}}: \mathrm{H} \rightarrow \mathrm{C}$ is a mapping defined by

$$
\mathrm{K}_{\mathrm{r}_{\mathrm{t}}} x=\left\{u \in C: \Theta(u, y)+\langle B u, y-u\rangle+\varphi(y)-\varphi(u)+\frac{1}{r_{t}}\langle y-u, u-x\rangle \geqslant 0, \forall y \in C\right\}
$$

for all $x \in H$ and for $r_{t} \in(0, \infty), t \in(0,1)$, and $\liminf _{t \rightarrow 0} r_{t}>0$;

- $\mathrm{F}_{\mathrm{r}_{\mathrm{t}}}: \mathrm{H} \rightarrow \mathrm{C}$ is a mapping defined by

$$
\mathrm{F}_{\mathrm{r}_{\mathrm{t}}} \mathrm{x}=\left\{z \in \mathrm{C}:\langle\mathrm{y}-z, \mathrm{~F} z\rangle+\frac{1}{\mathrm{r}_{\mathrm{t}}}\langle\mathrm{y}-z, z-x\rangle \geqslant 0, \quad \forall \mathrm{y} \in \mathrm{C}\right\}
$$

for $r_{t} \in(0, \infty), t \in(0,1)$, and $\liminf _{t \rightarrow 0} r_{t}>0$;

- $\mathrm{T}_{r_{t}}: \mathrm{H} \rightarrow \mathrm{C}$ is a mapping defined by

$$
\mathrm{T}_{\mathrm{r}_{\mathrm{t}}} x=\left\{z \in \mathrm{C}:\langle y-z, \mathrm{~T} z\rangle-\frac{1}{\mathrm{r}_{\mathrm{t}}}\left\langle y-z,\left(1+\mathrm{r}_{\mathrm{t}}\right) z-x\right\rangle \leqslant 0, \quad \forall \mathrm{y} \in \mathrm{C}\right\}
$$

for $r_{t} \in(0, \infty), t \in(0,1)$, and $\liminf _{t \rightarrow 0} r_{t}>0$;

- $\mathrm{K}_{\mathrm{r}_{\mathrm{n}}}: \mathrm{H} \rightarrow \mathrm{C}$ is a mapping defined by

$$
\mathrm{K}_{\mathrm{r}_{\mathrm{n}}} x=\left\{\mathrm{u} \in \mathrm{C}: \Theta(\mathrm{u}, \mathrm{y})+\langle\mathrm{Bu}, \mathrm{y}-\mathrm{u}\rangle+\varphi(\mathrm{y})-\varphi(\mathrm{u})+\frac{1}{\mathrm{r}_{\mathrm{n}}}\langle\mathrm{y}-\mathrm{u}, \mathrm{u}-\mathrm{x}\rangle \geqslant 0, \forall \mathrm{y} \in \mathrm{C}\right\}
$$

for all $x \in H$ and for $r_{n} \in(0, \infty)$ and $\liminf _{n \rightarrow \infty} r_{n}>0$; 
- $\mathrm{F}_{\mathrm{r}_{\mathrm{n}}}: \mathrm{H} \rightarrow \mathrm{C}$ is a mapping defined by

$$
\mathrm{F}_{\mathrm{r}_{\mathrm{n}}} x=\left\{z \in \mathrm{C}:\langle\mathrm{y}-z, \mathrm{~F} z\rangle+\frac{1}{\mathrm{r}_{\mathrm{n}}}\langle\mathrm{y}-z, z-x\rangle \geqslant 0, \quad \forall \mathrm{y} \in \mathrm{C}\right\}
$$

for $r_{n} \in(0, \infty)$ and $\liminf _{n \rightarrow \infty} r_{n}>0$;

- $\mathrm{T}_{\mathrm{r}_{\mathrm{n}}}: \mathrm{H} \rightarrow \mathrm{C}$ is a mapping defined by

$$
\mathrm{T}_{\mathrm{r}_{\mathrm{n}}} x=\left\{z \in \mathrm{C}:\langle\mathrm{y}-z, \mathrm{~T} z\rangle-\frac{1}{\mathrm{r}_{\mathrm{n}}}\left\langle\mathrm{y}-z,\left(1+\mathrm{r}_{\mathrm{n}}\right) z-x\right\rangle \leqslant 0, \quad \forall \mathrm{y} \in \mathrm{C}\right\}
$$

for $r_{n} \in(0, \infty)$ and $\liminf _{n \rightarrow \infty} r_{n}>0$;

- $\mathrm{V}: \mathrm{C} \rightarrow \mathrm{C}$ is l-Lipschitzian with constant $\mathrm{l} \in[0, \infty)$;

- $\mathrm{G}: \mathrm{C} \rightarrow \mathrm{C}$ is a $\rho$-Lipschitzian and $\eta$-strongly monotone mapping with constants $\rho>0$ and $\eta>0$;

- constants $\mu, l, \tau$, and $\gamma$ satisfy $0<\mu<\frac{2 \eta}{\rho^{2}}$ and $0 \leqslant \gamma l<\tau$, where $\tau=1-\sqrt{1-\mu\left(2 \eta-\mu \rho^{2}\right)}$;

- $\operatorname{GMEP}(\Theta, \varphi, \mathrm{B})$ is the set of solutions of the GMEP (1.1);

- $\operatorname{VI}(C, F)$ is the set of the variational inequality problem (1.5) for F;

- $\Omega:=\operatorname{GMEP}(\Theta, \varphi, \mathrm{B}) \cap \operatorname{VI}(\mathrm{C}, \mathrm{F}) \cap \operatorname{Fix}(\mathrm{T}) \neq \emptyset$.

By Lemmas 2.1, 2.2 and 2.3, $\mathrm{K}_{r_{t}}, F_{r_{t}}, T_{r_{t}}, K_{r_{n}}, F_{r_{n}}$ and $T_{r_{n}}$ are nonexpansive and Fix $\left(K_{r_{t}}\right)=\operatorname{GMEP}(\Theta, \varphi, B)$ $=\operatorname{Fix}\left(K_{r_{n}}\right), \operatorname{VI}(C, F)=\operatorname{Fix}\left(F_{r_{t}}\right)=\operatorname{Fix}\left(F_{r_{n}}\right)$, and $\operatorname{Fix}(T)=\operatorname{Fix}\left(T_{r_{t}}\right)=\operatorname{Fix}\left(T_{r_{n}}\right)$.

In this section, first we introduce the following modified hybrid iterative method that generates a net $\left\{x_{\mathbf{t}}\right\}_{t \in(0,1)}$ in an implicit way:

$$
\left\{\begin{array}{l}
\Theta\left(u_{t}, y\right)+\left\langle B u_{t}, y-u_{t}\right\rangle+\varphi(y)-\varphi\left(u_{t}\right)+\frac{1}{r_{t}}\left\langle y-u_{t}, u_{t}-x_{t}\right\rangle \geqslant 0, \quad \forall y \in C, \\
x_{t}=t \gamma V x_{t}+(I-t \mu G)\left(\theta_{t} x_{t}+\left(1-\theta_{t}\right) T_{r_{t}} F_{r_{t}} K_{r_{t}} x_{t}\right), \quad \forall t \in(0,1),
\end{array}\right.
$$

where $r_{t}>0$ for $t \in(0,1), \liminf _{t \rightarrow 0} r_{t}>0, \theta_{t} \in(0,1)$ for $t \in(0,1)$, and $0<\liminf _{t \rightarrow 0} \theta_{t} \leqslant$ $\lim \sup _{t \rightarrow 0} \theta_{t}<1$.

Consider the following mapping $Q_{t}$ on $C$ defined by

$$
Q_{t} x=t \gamma V x+(I-t \mu G)\left(\theta_{t} x+\left(1-\theta_{t}\right) T_{r_{t}} F_{r_{t}} K_{r_{t}} x\right)
$$

Let $R_{t} x=\theta_{t} x+\left(1-\theta_{t}\right) T_{r_{t}} F_{r_{t}} K_{r_{t}} x$. Since $T_{r_{t}}, F_{r_{t}}$, and $K_{r_{t}}$ are nonexpansive, we have for $x, z \in C$,

$$
\left\|\mathrm{R}_{\mathrm{t}} x-\mathrm{R}_{\mathrm{t}} z\right\| \leqslant \theta_{\mathrm{t}}\|x-z\|+\left(1-\theta_{\mathrm{t}}\right)\|x-z\|=\|x-z\| .
$$

So, from Lemma 2.8 , we derive

$$
\begin{aligned}
\left\|Q_{t} x-Q_{t} z\right\| & \leqslant t \gamma\|V x-V z\|+\left\|(I-t \mu G) R_{t} x-(I-t \mu G) R_{t} z\right\| \\
& \leqslant t \gamma l\|x-z\|+(1-t \tau)\|x-z\| \\
& =(1-t(\tau-\gamma l))\|x-z\| .
\end{aligned}
$$

Since $0<1-t(\tau-\gamma l)<1, Q_{t}$ is a contraction. Therefore, by the Banach contraction principle, $Q_{t}$ has a unique fixed point $x_{t} \in C$, which uniquely solves the fixed point equation

$$
x_{t}=t \gamma V x_{t}+(I-t \mu G)\left(\theta_{t} x_{t}+\left(1-\theta_{t}\right) T_{r_{t}} F_{r_{t}} K_{r_{t}} x_{t}\right) .
$$

Now, we establish the strong convergence of the net $\left\{x_{t}\right\}$ generated by (3.1) and show the existence of the $\mathrm{q} \in \Omega$, which solves the variational inequality (3.2) below.

Theorem 3.1. The nets $\left\{x_{t}\right\}$ and $\left\{u_{t}\right\}$ defined via (3.1) converge strongly, as $t \rightarrow 0$, to a point $q \in \Omega$, which is the unique solution of the following variational inequality:

$$
\langle(\mu \mathrm{G}-\gamma \mathrm{V}) \mathrm{q}, \mathrm{p}-\mathrm{q}\rangle \geqslant 0, \quad \forall \mathrm{p} \in \Omega .
$$


Proof. First, we can show easily the uniqueness of a solution of the variational inequality (3.2). In fact, noting that $0 \leqslant \gamma l<\tau$ and $\mu \eta \geqslant \tau \Leftrightarrow \rho \geqslant \eta$, it follows from Lemma 2.7 that

$$
\langle(\mu \mathrm{G}-\gamma \mathrm{V}) x-(\mu \mathrm{G}-\gamma \mathrm{V}) \mathrm{y}, x-y\rangle \geqslant(\mu \eta-\gamma l)\|x-y\|^{2} .
$$

That is, $\mu \mathrm{G}-\gamma \mathrm{V}$ is strongly monotone for $0 \leqslant \gamma l<\tau \leqslant \mu \eta$. So the variational inequality (3.2) has only one solution. Below we use $\mathrm{q} \in \Omega$ to denote the unique solution of the variational inequality (3.2).

By Lemma 2.1, we know that $u_{t}=K_{r_{t}} x_{t}$. From now, we put $z_{t}:=F_{r_{t}} u_{t}, w_{t}:=T_{r_{t}} z_{t}$ and $y_{t}:=$ $\theta_{t} x_{t}+\left(1-\theta_{t}\right) T_{r_{t}} F_{r_{t}} K_{r_{t}} x_{t}\left(=\theta_{t} x_{t}+\left(1-\theta_{t}\right) T_{t} z_{t}\right)$ for $t \in(0,1)$.

We divide the proof into several steps.

Step 1. We show that $\left\{x_{t}\right\}$ is bounded. To this end, take $p \in \Omega$. Then, from Lemma 2.1 (4), Lemma 2.2 (iii) and Lemma 2.3 (iii), it follows that $T_{r_{t}} p=p, F_{r_{t}} p=p$ and $p=K_{r_{t}} p$. Since $K_{r_{t}}$ is nonexpansive, we have

$$
\left\|u_{t}-p\right\|^{2}=\left\|K_{r_{t}} x_{t}-K_{r_{t}} p\right\|^{2} \leqslant\left\|x_{t}-p\right\|^{2}
$$

that is, $\left\|u_{t}-p\right\| \leqslant\left\|x_{t}-p\right\|$. Also

$$
\begin{aligned}
\left\|z_{t}-p\right\| & \leqslant\left\|F_{r_{t}} u_{t}-F_{r_{t}} p\right\| \\
& \leqslant\left\|u_{t}-p\right\| \leqslant\left\|x_{t}-p\right\| .
\end{aligned}
$$

It follows from (3.3) that

$$
\begin{aligned}
\left\|y_{t}-p\right\| & \leqslant \theta_{t}\left\|x_{t}-p\right\|+\left(1-\theta_{t}\right)\left\|T_{r_{t}} F_{r_{t}} K_{r_{t}} x_{t}-p\right\| \\
& \leqslant \theta_{t}\left\|x_{t}-p\right\|+\left(1-\theta_{t}\right)\left\|x_{t}-p\right\| \\
& \leqslant\left\|x_{t}-p\right\| .
\end{aligned}
$$

Therefore it follows from (3.4) and Lemma 2.8 that

$$
\begin{aligned}
\left\|x_{t}-p\right\| & =\left\|t \gamma V x_{t}+(I-t \mu G) y_{t}-p\right\| \\
& =\left\|t\left(\gamma \vee x_{t}-\gamma V p\right)+(I-t \mu G) y_{t}-(I-t \mu G) p+t(\gamma V p-\mu G p)\right\| \\
& \leqslant t \gamma l\left\|x_{t}-p\right\|+(1-t \tau)\left\|y_{t}-p\right\|+t\|\gamma V p-\mu G p\| \\
& \leqslant t \gamma l\left\|x_{t}-p\right\|+(1-t \tau)\left\|x_{t}-p\right\|+t(\gamma\|V p\|+\mu\|G p\|) .
\end{aligned}
$$

So, we derive

$$
\left\|x_{t}-p\right\| \leqslant \frac{\gamma\|V p\|+\mu\|G p\|}{\tau-\gamma l} .
$$

Thus, $\left\{x_{\mathfrak{t}}\right\}$ is bounded, and $\left\{\mathfrak{u}_{\mathfrak{t}}\right\},\left\{\mathrm{y}_{\mathrm{t}}\right\},\left\{\mathrm{G} \mathrm{y}_{\mathrm{t}}\right\},\left\{z_{\mathrm{t}}\right\},\left\{\mathrm{V} \mathrm{x}_{\mathrm{t}}\right\}$ and $\left\{\mathrm{F} \mathfrak{u}_{\mathrm{t}}\right\}$ are also bounded.

Step 2. We show that $\lim _{t \rightarrow 0}\left\|x_{t}-w_{t}\right\|=\lim _{t \rightarrow 0}\left\|x_{t}-T_{r_{t}} z_{t}\right\|=0$. In fact, observing

$$
\begin{aligned}
\left\|x_{\mathrm{t}}-\mathrm{T}_{\mathrm{r}_{\mathrm{t}}} z_{\mathrm{t}}\right\| & =\left\|\mathrm{t} \gamma \mathrm{V} x_{\mathrm{t}}+(\mathrm{I}-\mathrm{t} \mu \mathrm{G}) \mathrm{y}_{\mathrm{t}}-\mathrm{T}_{\mathrm{r}_{\mathrm{t}}} z_{\mathrm{t}}\right\| \\
& \leqslant \mathrm{t}\left\|\gamma \mathrm{V} x_{\mathrm{t}}-\mu \mathrm{G} y_{\mathrm{t}}\right\|+\left\|y_{\mathrm{t}}-\mathrm{T}_{\mathrm{r}_{\mathrm{t}}} z_{\mathrm{t}}\right\| \\
& =\mathrm{t}\left\|\gamma \mathrm{V} x_{\mathrm{t}}-\mu \mathrm{G} y_{\mathrm{t}}\right\|+\left\|\theta_{\mathrm{t}} x_{\mathrm{t}}+\left(1-\theta_{\mathrm{t}}\right) \mathrm{T}_{\mathrm{r}_{\mathrm{t}}} z_{\mathrm{t}}-\mathrm{T}_{\mathrm{r}_{\mathrm{t}}} z_{\mathrm{t}}\right\| \\
& =\mathrm{t}\left\|\gamma \mathrm{V} x_{\mathrm{t}}-\mu G y_{\mathrm{t}}\right\|+\theta_{\mathrm{t}}\left\|\mathrm{x}_{\mathrm{t}}-\mathrm{T}_{\mathrm{r}_{\mathrm{t}}} z_{\mathrm{t}}\right\|,
\end{aligned}
$$

we have

$$
\left\|x_{\mathrm{t}}-\mathrm{T}_{\mathrm{r}_{\mathrm{t}}} z_{\mathrm{t}}\right\| \leqslant \frac{\mathrm{t}}{1-\theta_{\mathrm{t}}}\left\|\gamma \mathrm{V} \mathrm{x}_{\mathrm{t}}-\mu \mathrm{G} \mathrm{y}_{\mathrm{t}}\right\| \rightarrow 0 \text { as } \mathrm{t} \rightarrow 0 .
$$

Step 3. We show that $\lim _{t \rightarrow 0}\left\|x_{t}-u_{t}\right\|=0$. To this end, let $p \in \Omega$. Since $K_{r_{t}}$ is firmly nonexpansive and $u_{t}=k_{r_{t}} x_{t}$, we have

$$
\left\|u_{t}-p\right\|^{2}=\left\|K_{r_{t}} x_{t}-K_{r_{t}} p\right\|^{2}
$$




$$
\begin{aligned}
& \leqslant\left\langle K_{r_{t}} x_{t}-K_{r_{t}} p, x_{t}-x_{t}-p\right\rangle \\
& =\frac{1}{2}\left(\left\|u_{t}-p\right\|^{2}+\left\|x_{t}-p\right\|^{2}\right)-\frac{1}{2}\left(\left\|x_{t}-p-\left(u_{t}-p\right)\right\|^{2}\right) \\
& =\frac{1}{2}\left(\left\|u_{t}-p\right\|^{2}+\left\|x_{t}-p\right\|^{2}-\left\|x_{t}-u_{t}\right\|^{2}\right)
\end{aligned}
$$

and hence

$$
\left\|u_{t}-p\right\|^{2} \leqslant\left\|x_{t}-p\right\|^{2}-\left\|x_{t}-u_{t}\right\|^{2} .
$$

Moreover, from $z_{t}=F_{r_{t}} u_{t}$, we get

$$
\left\|z_{t}-p\right\|^{2}=\left\|F_{r_{t}} u_{t}-F_{r_{t}} p\right\|^{2} \leqslant\left\|u_{t}-p\right\|^{2} .
$$

By (3.5) and (3.6), we obtain

$$
\begin{aligned}
\left\|x_{t}-p\right\|^{2}= & \left\|t\left(\gamma \vee x_{t}-\mu G y_{t}\right)+\left(y_{t}-p\right)\right\|^{2} \\
= & \left\|t\left(\gamma \vee x_{t}-\mu G y_{t}\right)+\theta_{t}\left(x_{t}-T_{r_{t}} z_{t}\right)+\left(T_{r_{t}} z_{t}-p\right)\right\|^{2} \\
\leqslant & {\left[\left(\left\|t\left(\gamma \vee x_{t}-\mu G y_{t}\right)\right\|+\left\|z_{t}-p\right\|\right)+\theta_{t}\left\|x_{t}-T_{r_{t}} z_{t}\right\|\right]^{2} } \\
= & t^{2}\left\|\gamma \vee x_{t}-\mu G y_{t}\right\|^{2}+2 t\left\|\gamma \vee x_{t}-\mu G y_{t}\right\|\left\|z_{t}-p\right\|+\left\|z_{t}-p\right\|^{2} \\
& \quad+\theta_{t}\left\|x_{t}-T_{r_{t}} z_{t}\right\|\left[2\left(t\left\|\gamma \vee x_{t}-\mu G y_{t}\right\|+\left\|z_{t}-p\right\|\right)+\theta_{t}\left\|x_{t}-T_{r_{t}} z_{t}\right\|\right] \\
\leqslant & t\left\|\gamma \vee x_{t}-\mu G y_{t}\right\|^{2}+\left\|u_{t}-p\right\|^{2}+M_{t} \\
\leqslant & t\left\|\gamma V x_{t}-\mu G y_{t}\right\|^{2}+\left(\left\|x_{t}-p\right\|^{2}-\left\|x_{t}-u_{t}\right\|^{2}\right)+M_{t}
\end{aligned}
$$

where

$$
M_{t}=\theta_{t}\left\|x_{t}-T_{r_{t}} z_{t}\right\|\left[2\left(t\left\|\gamma V x_{t}-\mu G y_{t}\right\|+\left\|z_{t}-p\right\|\right)+\theta_{t}\left\|x_{t}-T_{r_{t}} z_{t}\right\|\right]+2 t\left\|\gamma V x_{t}-\mu G y_{t}\right\|\left\|z_{t}-p\right\| .
$$

Now, from (3.7), we derive

$$
\left\|x_{t}-u_{t}\right\|^{2} \leqslant t\left\|\gamma V x_{t}-\mu G y_{t}\right\|^{2}+M_{t} .
$$

Since $M_{t} \rightarrow 0$ by Step 2, we have

$$
\lim _{t \rightarrow 0}\left\|x_{t}-u_{t}\right\|=0 .
$$

Step 4. We show that $\lim _{t \rightarrow 0}\left\|u_{t}-z_{t}\right\|=0$. To this end, let $p \in \Omega$. Using $z_{t}=F_{r_{t}} u_{t}$ and $p=F_{r_{t}} p$, we obtain

$$
\begin{aligned}
\left\|z_{t}-p\right\|^{2} & =\left\|F_{r_{t}} u_{t}-F_{r_{t}} p\right\|^{2} \\
& \leqslant\left\langle F_{r_{t}} u_{t}-F_{r_{t}} p, u_{t}-p\right\rangle \\
& =\left\langle z_{t}-p, u_{t}-p\right\rangle \\
& \leqslant \frac{1}{2}\left[\left\|z_{t}-p\right\|^{2}+\left\|u_{t}-p\right\|^{2}-\left\|u_{t}-z_{t}\right\|^{2}\right],
\end{aligned}
$$

that is,

$$
\begin{aligned}
\left\|z_{t}-p\right\|^{2} & \leqslant\left\|u_{t}-p\right\|^{2}-\left\|u_{t}-z_{t}\right\|^{2} \\
& \leqslant\left\|x_{t}-p\right\|^{2}-\left\|u_{t}-z_{t}\right\|^{2} .
\end{aligned}
$$

Thus, from (3.7) and (3.9), we deduce

$$
\begin{aligned}
\left\|x_{t}-p\right\|^{2} & \leqslant t\left\|\gamma V x_{t}-\mu G y_{t}\right\|^{2}+\left\|z_{t}-p\right\|^{2}+M_{t} \\
& \leqslant t\left\|\gamma V x_{t}-\mu G y_{t}\right\|^{2}+\left\|x_{t}-p\right\|^{2}-\left\|u_{t}-z_{t}\right\|^{2}+M_{t},
\end{aligned}
$$

which implies that

$$
\left\|u_{t}-z_{t}\right\|^{2} \leqslant t\left\|\gamma V x_{t}-\mu G y_{t}\right\|^{2}+M_{t},
$$


where $M_{t}$ is of in (3.8). From $\lim _{t \rightarrow 0} M_{t}=0$, it follows that

$$
\lim _{\mathrm{t} \rightarrow 0}\left\|\mathrm{u}_{\mathrm{t}}-z_{\mathrm{t}}\right\|=0 \text {. }
$$

Step 5. We show that $\lim _{\mathrm{t} \rightarrow 0}\left\|z_{\mathrm{t}}-w_{\mathrm{t}}\right\|=\lim _{\mathrm{t} \rightarrow 0}\left\|z_{\mathrm{t}}-\mathrm{T}_{\mathrm{r}_{\mathrm{t}}} z_{\mathrm{t}}\right\|=0$. In fact, since $\left\|w_{\mathrm{t}}-z_{\mathrm{t}}\right\|=\left\|\mathrm{T}_{\mathrm{r}_{\mathrm{t}}} z_{\mathrm{t}}-z_{\mathrm{t}}\right\| \leqslant$ $\left\|\mathrm{T}_{\mathrm{r}_{\mathrm{t}}} z_{\mathrm{t}}-\mathrm{x}_{\mathrm{t}}\right\|+\left\|\mathrm{x}_{\mathrm{t}}-\mathrm{u}_{\mathrm{t}}\right\|+\left\|\mathrm{u}_{\mathrm{t}}-z_{\mathrm{t}}\right\|$, by Step 2, Step 3 and Step 4, we conclude that

$$
\lim _{\mathrm{t} \rightarrow 0}\left\|w_{\mathrm{t}}-z_{\mathrm{t}}\right\|=0 \text {. }
$$

Step 6. We show that $\lim _{\mathrm{t} \rightarrow 0}\left\|x_{\mathrm{t}}-z_{\mathrm{t}}\right\|=0$. In fact, from Step 2 and Step 4, it follows that

$$
\left\|x_{\mathrm{t}}-z_{\mathrm{t}}\right\| \leqslant\left\|x_{\mathrm{t}}-\mathrm{T}_{\mathrm{r}_{\mathrm{t}}} z_{\mathrm{t}}\right\|+\left\|\mathrm{T}_{\mathrm{r}_{\mathrm{t}}} z_{\mathrm{t}}-z_{\mathrm{t}}\right\| \rightarrow 0 \text { as } \mathrm{t} \rightarrow 0 \text {. }
$$

Step 7. We show that $\left\{x_{t}\right\}$ is relatively norm compact as $t \rightarrow 0$. To this end, let $\left\{t_{n}\right\} \subset(0,1)$ be a sequence such that $t_{n} \rightarrow 0$ as $n \rightarrow \infty$. Put $x_{n}:=x_{t_{n}}, u_{n}:=u_{t_{n}}, z_{n}:=z_{t_{n}}, w_{n}:=w_{t_{n}}$ and $r_{n}:=r_{t_{n}}$. First of all, by (3.1), we deduce

$$
\begin{aligned}
\left\|x_{t}-p\right\|^{2}= & \left\|t \gamma V x_{t}+(I-t \mu G) y_{t}-p\right\|^{2} \\
= & \left\|(I-t \mu G) y_{t}-(I-t \mu G) p-t(\mu G-\gamma V) p+t \gamma\left(V x_{t}-V p\right)\right\|^{2} \\
= & \left\|(I-t \mu G) y_{t}-(I-t \mu G) p\right\|^{2}-2 t\left[\left\langle(\mu G-\gamma V) p, y_{t}-p\right\rangle-t\left\langle(\mu G-\gamma V) p, \mu G y_{t}-\mu G p\right\rangle\right] \\
& +2 t \gamma\left[\left\langle V x_{t}-V p, y_{t}-p\right\rangle-t\left\langle V x_{t}-V p, \mu G y_{t}-\mu G p\right\rangle\right] \\
& -2 t^{2} \gamma\left\langle(\mu G-\gamma V) p, V x_{t}-V p\right\rangle+t^{2}\|(\mu G-\gamma V) p\|^{2}+t^{2} \gamma^{2}\left\|V x_{t}-V p\right\|^{2} \\
\leqslant & (1-t \tau)^{2}\left\|y_{t}-p\right\|^{2}-2 t\left\langle(\mu G-\gamma V) p, y_{t}-p\right\rangle+2 t \gamma l\left\|x_{t}-p\right\|\left\|y_{t}-p\right\| \\
& +2 t^{2}\|(\mu G-\gamma V) p\|\left(\left\|\mu G y_{t}\right\|+\|\mu G p\|\right) \\
& +2 t^{2} \gamma l\left\|x_{t}-p\right\|\left(\left\|\mu G y_{t}\right\|+\|\mu G p\|\right)+2 t^{2} l\|(\mu G-\gamma V) p\|\left\|x_{t}-p\right\| \\
& +t^{2}\left(\|(\mu G-V) p\|^{2}+\gamma^{2} l^{2}\left\|x_{t}-p\right\|^{2}\right) \\
= & \left(1-2 t \tau+t^{2} \tau^{2}\right)\left\|y_{t}-p\right\|^{2}-2 t\left\langle(\mu G-\gamma V) p, y_{t}-p\right\rangle \\
& +2 t \gamma l\left\|x_{t}-p\right\|\left\|y_{t}-p\right\|+2 t^{2}\|(\mu G-\gamma V) p\|\left(\left\|(\mu G) y_{t}\right\|+\|\mu G p\|\right) \\
& +2 t^{2} \gamma l\left\|x_{t}-p\right\|\left(\left\|\mu G y_{t}\right\|+\|\mu G p\|\right)+2 t^{2} l\|(\mu G-\gamma V) p\|\left\|x_{t}-p\right\| \\
& +t^{2}\left(\|(\mu G-V) p\|^{2}+\gamma^{2} l^{2}\left\|x_{t}-p\right\|^{2}\right) \\
\leqslant & (1-2 t \tau)\left\|y_{t}-p\right\|^{2}+2 t\left\langle(\mu G-\gamma V) p, p-y_{t}\right\rangle+t \gamma l\left(\left\|x_{t}-p\right\|^{2}+\left\|y_{t}-p\right\|^{2}\right)+t^{2} M,
\end{aligned}
$$

where

$$
\begin{aligned}
M=\sup \left\{\tau^{2}\left\|y_{t}-p\right\|^{2}+2\left(\|(\mu G-\gamma V) p\|+\gamma l \mu\left\|x_{t}-p\right\|\right)\left(\left\|G y_{t}\right\|+\|G p\|\right)\right. \\
\left.+2 l\|(\mu G-\gamma V) p\|\left\|x_{t}-p\right\|+\|(\mu G-\gamma V) p\|^{2}+\gamma^{2} l^{2}\left\|x_{t}-p\right\|^{2}\right\} .
\end{aligned}
$$

Hence, for small enough $t$, by (3.4), we obtain

$$
\begin{aligned}
\left\|x_{t}-p\right\|^{2} & \leqslant \frac{1-2 t \tau+t \gamma l}{1-t \gamma l}\left\|y_{t}-p\right\|^{2}+\frac{2 t}{1-t \gamma l}\left\langle(\mu G-\gamma V) p, p-y_{t}\right\rangle+\frac{t^{2}}{1-t \gamma l} M \\
& \leqslant \frac{1-2 t \tau+t \gamma l}{1-t \gamma l}\left\|x_{t}-p\right\|^{2}+\frac{2 t}{1-t \gamma l}\left\langle(\mu G-\gamma V) p, p-y_{t}\right\rangle+\frac{t^{2}}{1-t \gamma l} M .
\end{aligned}
$$

Observe that

$$
\begin{aligned}
\left\langle(\mu \mathrm{G}-\gamma \mathrm{V}) \mathrm{p}, \mathrm{p}-\mathrm{y}_{\mathrm{t}}\right\rangle= & \left\langle(\mu \mathrm{G}-\gamma \mathrm{V}) \mathrm{p}, \mathrm{p}-\left(\theta_{\mathrm{t}} x_{\mathrm{t}}+\left(1-\theta_{\mathrm{t}}\right) \mathrm{T}_{\mathrm{r}_{\mathrm{t}}} z_{\mathrm{t}}\right)\right\rangle \\
= & \left\langle(\mu \mathrm{G}-\gamma \mathrm{V}) \mathrm{p}, \mathrm{p}-\mathrm{T}_{\mathrm{r}_{\mathrm{t}}} z_{\mathrm{t}}\right\rangle+\theta_{\mathrm{t}}\left\langle(\mu \mathrm{G}-\gamma \mathrm{V}) \mathrm{p}, \mathrm{T}_{\mathrm{r}_{\mathrm{t}}} z_{\mathrm{t}}-x_{\mathrm{t}}\right\rangle \\
= & \left\langle(\mu \mathrm{G}-\gamma \mathrm{V}) \mathrm{p}, \mathrm{p}-z_{\mathrm{t}}\right\rangle+\left\langle(\mu \mathrm{G}-\gamma \mathrm{V}) \mathrm{p}, z_{\mathrm{t}}-\mathrm{T}_{\mathrm{r}_{\mathrm{t}}} z_{\mathrm{t}}\right\rangle \\
& +\theta_{\mathrm{t}}\left\langle(\mu \mathrm{G}-\gamma \mathrm{V}) \mathrm{p}, \mathrm{T}_{\mathrm{r}_{\mathrm{t}}} z_{\mathrm{t}}-x_{\mathrm{t}}\right\rangle \\
= & \left\langle(\mu \mathrm{G}-\gamma \mathrm{V}) \mathrm{p}, \mathrm{p}-z_{\mathrm{t}}\right\rangle+\left\langle(\mu \mathrm{G}-\gamma \mathrm{V}) \mathrm{p}, z_{\mathrm{t}}-\mathrm{T}_{\mathrm{r}_{\mathrm{t}}} z_{\mathrm{t}}\right\rangle \\
& +\theta_{\mathrm{t}}\left\langle(\mu \mathrm{G}-\gamma \mathrm{V}) \mathrm{p}, \mathrm{T}_{\mathrm{r}_{\mathrm{t}}} z_{\mathrm{t}}-x_{\mathrm{t}}\right\rangle \\
\leqslant & \left\langle(\mu \mathrm{G}-\gamma \mathrm{V}) \mathrm{p}, \mathrm{p}-z_{\mathrm{t}}\right\rangle+\|(\mu \mathrm{G}-\gamma \mathrm{V}) \mathrm{p}\|\left\|z_{\mathrm{t}}-\mathrm{T}_{\mathrm{r}_{\mathrm{t}}} z_{\mathrm{t}}\right\| \\
& +\theta_{\mathrm{t}}\|(\mu \mathrm{G}-\gamma \mathrm{V}) \mathrm{p}\|\left\|\mathrm{T}_{\mathrm{r}_{\mathrm{t}}} z_{\mathrm{t}}-x_{\mathrm{t}}\right\| \\
\leqslant & \left\langle(\mu \mathrm{G}-\gamma \mathrm{V}) \mathrm{p}, \mathrm{p}-z_{\mathrm{t}}\right\rangle+\mathrm{L}_{\mathrm{t}},
\end{aligned}
$$


where $\mathrm{L}_{\mathrm{t}}=\sup \left\{\|(\mu \mathrm{G}-\gamma \mathrm{V}) \mathrm{p}\|\left\|z_{\mathrm{t}}-\mathrm{T}_{\mathrm{r}_{\mathrm{t}}} z_{\mathrm{t}}\right\|+\theta_{\mathrm{t}}\|(\mu \mathrm{G}-\gamma \mathrm{V}) \mathrm{p}\|\left\|\mathrm{T}_{\mathrm{r}_{\mathrm{t}}} z_{\mathrm{t}}-x_{\mathrm{t}}\right\|\right\}$. Then, from (3.10) and (3.11), we derive that

$$
\left\|x_{t}-p\right\|^{2} \leqslant \frac{1}{\tau-\gamma l}\left\langle(\mu G-\gamma V) p, p-z_{t}\right\rangle+\frac{t M}{2(\tau-\gamma l)}+\frac{L_{t}}{\tau-\gamma l} .
$$

In particular,

$$
\left\|x_{n}-p\right\|^{2} \leqslant \frac{1}{\tau-\gamma l}\left\langle(\mu G-\gamma V) p, p-z_{n}\right\rangle+\frac{t_{n} M}{2(\tau-\gamma l)}+\frac{L_{t_{n}}}{\tau-\gamma l} .
$$

Since $\left\{x_{n}\right\}$ is bounded, without loss of generality, we may assume that $\left\{x_{n}\right\}$ converges weakly to a point $q \in C$. Then, by the same argument as in Step 6 in the proof of [15, Theorem 3.1], we can show that $\mathrm{q} \in \Omega$. For the sake of completeness, we include its proof.

First, we show that $q \in \operatorname{GMEP}(\Theta, \varphi, B)$. Indeed, by $u_{n}=K_{r_{n}} x_{n}$, we know that

$$
\Theta\left(u_{n}, y\right)+\left\langle B u_{n}, y-u_{n}\right\rangle+\varphi(y)-\varphi\left(u_{n}\right)+\frac{1}{r_{n}}\left\langle y-u_{n}, u_{n}-x_{n}\right\rangle \geqslant 0, \quad \forall y \in C .
$$

It follows from (A2) that

$$
\left\langle B u_{n}, y-u_{n}\right\rangle+\varphi(y)-\varphi\left(u_{n}\right)+\frac{1}{r_{n}}\left\langle y-u_{n}, u_{n}-x_{n}\right\rangle \geqslant \Theta\left(y, u_{n}\right), \quad \forall y \in C .
$$

Hence

$$
\left\langle B u_{n}, y-u_{n}\right\rangle+\varphi(y)-\varphi\left(u_{n}\right)+\frac{1}{r_{n}}\left\langle y-u_{n}, u_{n}-x_{n}\right\rangle \geqslant \Theta\left(y, u_{n}\right), \quad \forall y \in C .
$$

For $\mathrm{t}$ with $0<\mathrm{t} \leqslant 1$ and $w \in \mathrm{C}$, let $v_{\mathrm{t}}=\mathrm{t} v+(1-\mathrm{t}) \mathrm{q}$. Since $v \in \mathrm{C}$ and $\mathrm{q} \in \mathrm{C}$, we have $w_{\mathrm{t}} \in \mathrm{C}$. So, from (3.13), we have

$$
\begin{aligned}
\left\langle B v_{t}, v_{t}-u_{n}\right\rangle & \geqslant\left\langle B v_{t}, v_{t}-u_{n}\right\rangle-\varphi\left(v_{t}\right)+\varphi\left(u_{n}\right)-\left\langle B u_{n}, v_{t}-u_{n}\right\rangle-\left\langle v_{t}-u_{n}, \frac{u_{n}-x_{n}}{r_{n}}\right\rangle+\Theta\left(v_{t}, u_{n}\right) \\
& =\left\langle B v_{t}-B u_{n}, v_{t}-u_{n}\right\rangle-\varphi\left(v_{t}\right)+\varphi\left(u_{n}\right)-\left\langle v_{t}-u_{n}, \frac{u_{n}-x_{n}}{r_{n}}\right\rangle+\Theta\left(v_{t}, u_{n}\right) .
\end{aligned}
$$

Since $\left\|u_{n}-x_{n}\right\| \rightarrow 0$ by Step $3, \frac{u_{n}-x_{n}}{r_{n}} \rightarrow 0$ and $u_{n} \rightarrow q$. Moreover, from the monotonicity of B, we have $\left\langle B v_{t}-B u_{n}, y_{t}-u_{n}\right\rangle \geqslant 0$. So, from (A4) and the weak lower semicontinuity of $\varphi$, if follows that

$$
\left\langle\mathrm{B} v_{\mathrm{t}}, v_{\mathrm{t}}-\mathrm{q}\right\rangle \geqslant-\varphi\left(v_{\mathrm{t}}\right)+\varphi(\mathrm{q})+\Theta\left(v_{\mathrm{t}}, \mathrm{q}\right) \text { as } i \rightarrow \infty
$$

By (A1), (A4) and (3.14), we also obtain

$$
\begin{aligned}
0 & =\Theta\left(v_{\mathrm{t}}, v_{\mathrm{t}}\right)+\varphi\left(v_{\mathrm{t}}\right)-\varphi\left(v_{\mathrm{t}}\right) \\
& \leqslant \mathrm{t} \Theta\left(v_{\mathrm{t}}, v\right)+(1-\mathrm{t}) \Theta\left(v_{\mathrm{t}}, \mathrm{q}\right)+\mathrm{t} \varphi(v)+(1-\mathrm{t}) \varphi(\mathrm{q})-\varphi\left(v_{\mathrm{t}}\right) \\
& \leqslant \mathrm{t}\left[\Theta\left(v_{\mathrm{t}}, v\right)+\varphi(v)-\varphi\left(v_{\mathrm{t}}\right)\right]+(1-\mathrm{t})\left\langle\mathrm{B} v_{\mathrm{t}}, v_{\mathrm{t}}-\mathrm{q}\right\rangle \\
& =\mathrm{t}\left[\Theta\left(v_{\mathrm{t}}, v\right)+\varphi(v)-\varphi\left(v_{\mathrm{t}}\right)\right]+(1-\mathrm{t}) \mathrm{t}\left\langle\mathrm{B} v_{\mathrm{t}}, v-\mathrm{q}\right\rangle,
\end{aligned}
$$

and hence

$$
0 \leqslant \Theta\left(v_{t}, v\right)+\varphi(v)-\varphi\left(v_{t}\right)+(1-t)\left\langle B v_{t}, v-q\right\rangle
$$

Letting $t \rightarrow 0$ in (3.15), we have for each $v \in C$

$$
\Theta(q, v)+\langle B q, v-q\rangle+\varphi(v)-\varphi(q) \geqslant 0 .
$$

This implies that $q \in \operatorname{GMEP}(\Theta, \varphi, \mathrm{B})$.

Second, we show that $q \in V I(C, F)$. In fact, from the definition of $z_{n}=F_{r_{n}} u_{n}$, we have

$$
\left\langle y-z_{n}, F z_{n}\right\rangle+\left\langle y-z_{n}, \frac{z_{n}-u_{n}}{r_{n}}\right\rangle \geqslant 0, \quad \forall y \in C .
$$


Set $v_{\mathrm{t}}=\mathrm{t} v+(1-\mathrm{t}) \mathrm{q}$, for all $\mathrm{t} \in(0,1]$ and $v \in \mathrm{C}$. Then, $v_{\mathrm{t}} \in \mathrm{C}$. From (3.16), it follows that

$$
\begin{aligned}
\left\langle v_{\mathrm{t}}-z_{\mathrm{n}}, \mathrm{F} v_{\mathrm{t}}\right\rangle & \geqslant\left\langle v_{\mathrm{t}}-z_{\mathrm{n}}, \mathrm{F} v_{\mathrm{t}}\right\rangle-\left\langle v_{\mathrm{t}}-z_{\mathrm{n}}, \mathrm{F} z_{\mathrm{n}}\right\rangle-\left\langle v_{\mathrm{t}}-z_{\mathrm{n}}, \frac{z_{\mathrm{n}}-\mathrm{u}_{\mathrm{n}}}{\mathrm{r}_{\mathrm{n}}}\right\rangle \\
& =\left\langle v_{\mathrm{t}}-z_{\mathrm{n}}, \mathrm{F} v_{\mathrm{t}}-\mathrm{F} z_{\mathrm{n}}\right\rangle-\left\langle v_{\mathrm{t}}-z_{\mathrm{n}}, \frac{z_{\mathrm{n}}-\mathrm{u}_{\mathrm{n}}}{r_{\mathrm{n}}}\right\rangle .
\end{aligned}
$$

By Step 4, we have $\frac{z_{\mathfrak{n}}-\mathfrak{u}_{n}}{r_{n}} \rightarrow 0$ as $\mathrm{n} \rightarrow \infty$. Moreover, since $x_{\mathfrak{n}} \rightarrow \mathrm{q}$, by Step 6, we have $z_{\mathfrak{n}} \rightarrow \mathrm{q}$ as $\mathrm{n} \rightarrow \infty$. Since $F$ is monotone, we also have that $\left\langle v_{t}-z_{n}, F v_{t}-F z_{n}\right\rangle \geqslant 0$. Thus, from (3.17), it follows that

$$
0 \leqslant \lim _{n \rightarrow \infty}\left\langle v_{t}-z_{n}, F v_{t}\right\rangle=\left\langle v_{t}-q, F v_{t}\right\rangle
$$

and hence

$$
\left\langle v-\mathrm{q}, \mathrm{F} v_{\mathrm{t}}\right\rangle \geqslant 0, \quad \forall v \in \mathrm{C} .
$$

If $t \rightarrow 0$, the continuity of $F$ yields that

$$
\langle v-\mathrm{q}, \mathrm{Fq}\rangle \geqslant 0, \quad \forall v \in \mathrm{C} .
$$

This implies that $q \in V I(C, F)$.

Third, we show that $q \in \operatorname{Fix}(T)$. In fact, from the definition of $w_{n}=T_{r_{n}} z_{n}$, we have

$$
\left\langle y-w_{n}, T w_{n}\right\rangle-\frac{1}{r_{n}}\left\langle y-w_{n},\left(1+r_{n}\right) w_{n}-z_{n}\right\rangle \leqslant 0, \quad \forall y \in C .
$$

Put $v_{\mathrm{t}}=\mathrm{t} v+(1-\mathrm{t}) \mathrm{q}$ for all $\mathrm{t} \in(0,1]$ and $v \in \mathrm{C}$. Then $v_{\mathrm{t}} \in \mathrm{C}$ and from (3.18) and pseudocontractivity of $\mathrm{T}$, it follows that

$$
\begin{aligned}
\left\langle w_{n}-v_{t}, T v_{t}\right\rangle & \geqslant\left\langle w_{n}-v_{t}, T v_{t}\right\rangle+\left\langle v_{t}-w_{n}, T w_{n}\right\rangle-\frac{1}{r_{n}}\left\langle v_{t}-w_{n},\left(1+r_{n}\right) w_{n}-z_{n}\right\rangle \\
& =-\left\langle v_{t}-w_{n}, T v_{t}-T w_{n}\right\rangle-\frac{1}{r_{n}}\left\langle v_{t}-u_{n}, w_{n}-z_{n}\right\rangle-\left\langle v_{t}-w_{n}, w_{n}\right\rangle \\
& \geqslant-\left\|v_{t}-w_{n}\right\|^{2}-\frac{1}{r_{n}}\left\langle v_{t}-w_{n}, w_{n}-z_{n}\right\rangle-\left\langle v_{t}-w_{n}, w_{n}\right\rangle \\
& =-\left\langle v_{t}-w_{n}, v_{t}\right\rangle-\left\langle v_{t}-w_{n}, \frac{w_{n}-z_{n}}{r_{n}}\right\rangle
\end{aligned}
$$

By Step 5, we get $\frac{w_{n}-z_{n}}{r_{n}} \rightarrow 0$ as $n \rightarrow \infty$. Moreover, since $x_{n} \rightarrow q$, by Step 2, we have $w_{n} \rightarrow q$ as $n \rightarrow \infty$. Therefore, from (3.19), as $n \rightarrow \infty$, it follows that

$$
\left\langle q-v_{t}, T v_{t}\right\rangle \geqslant\left\langle q-v_{t}, v_{t}\right\rangle
$$

and hence

$$
-\left\langle v-\mathrm{q}, \mathrm{T} v_{\mathrm{t}}\right\rangle \geqslant-\left\langle v-\mathrm{q}, v_{\mathrm{t}}\right\rangle, \quad \forall v \in \mathrm{C} .
$$

Letting $t \rightarrow 0$ and using the fact that $T$ is continuous, we get

$$
-\langle v-\mathrm{q}, \mathrm{Tq}\rangle \geqslant-\langle v-\mathrm{q}, \mathrm{q}\rangle, \quad \forall v \in \mathrm{C} .
$$

Now, let $v=T q$. Then we obtain $q=T q$ and hence $q \in \operatorname{Fix}(T)$. Therefore, $q \in \Omega$.

Now, we substitute $q$ for $p$ in (3.12) to obtain

$$
\left\|x_{n}-q\right\|^{2} \leqslant \frac{1}{\tau-\gamma l}\left\langle(\mu G-\gamma V) q, q-z_{n}\right\rangle+\frac{t_{n} M}{2(\tau-\gamma l)}+\frac{L_{t_{n}}}{\tau-\gamma l} .
$$

Note that $z_{n} \rightarrow q$ by Step 5 and $\lim _{t \rightarrow 0} L_{t}=0$ by Step 2 and Step 4 . This fact and the inequality (3.20) imply that $x_{n} \rightarrow q$ strongly. This has proved the relative norm compactness of the net $\left\{x_{t}\right\}$ as $t \rightarrow 0$. 
Step 8. We show that $q$ solves the variational inequality (3.2). In fact, taking the limit in (3.12) as $n \rightarrow \infty$, we get

$$
\|q-p\|^{2} \leqslant \frac{1}{\tau-\gamma l}\langle(\mu G-\gamma V) p, p-q\rangle, \quad \forall p \in \Omega .
$$

In particular, q solves the following variational inequality

$$
q \in \Omega, \quad\langle(\mu G-\gamma V) p, p-q\rangle \geqslant 0, \quad p \in \Omega,
$$

or the equivalent dual variational inequality (see [20])

$$
q \in \Omega, \quad\langle(\mu \mathrm{G}-\gamma \mathrm{V}) \mathrm{q}, \mathrm{p}-\mathrm{q}\rangle \geqslant 0, \quad \mathrm{p} \in \Omega .
$$

Step 9. We show that the entire net $\left\{x_{t}\right\}$ converges strongly to q. To this end, let $\left\{x_{n_{k}}\right\}$ be another subsequence of $\left\{x_{n}\right\}$ and assume $x_{n_{k}} \rightarrow \hat{q}$. By the same as the proof above, we have $\hat{q} \in \Omega$. Moreover, it follows from (3.21) that

$$
\langle(\mu \mathrm{G}-\gamma \mathrm{V}) \mathrm{q}, \hat{\mathrm{q}}-\mathrm{q}\rangle \geqslant 0
$$

Interchanging $q$ and $\hat{q}$, we obtain

$$
\langle(\mu \mathrm{G}-\gamma \mathrm{V}) \hat{\mathrm{q}}, \mathrm{q}-\hat{\mathrm{q}}\rangle \geqslant 0 .
$$

Lemma 2.7 and adding these two inequalities (3.22) and (3.23) yields

$$
(\mu \eta-\gamma l)\|q-\hat{q}\|^{2} \leqslant\langle(\mu G-\gamma V) q-(\mu G-\gamma V) \hat{q}, q-\hat{q}\rangle \leqslant 0 .
$$

Hence $q=\hat{q}$. Therefore we conclude that $x_{t} \rightarrow q$ as $t \rightarrow 0$. Moreover, by Step 3, we obtain that $u_{t} \rightarrow q$ as $t \rightarrow 0$.

From Theorem 3.1, we can deduce the following result.

Corollary 3.2. Let $\left\{x_{\mathrm{t}}\right\}$ and $\left\{\mathrm{u}_{\mathrm{t}}\right\}$ be nets generated by

$$
\left\{\begin{array}{l}
\Theta\left(u_{t}, y\right)+\left\langle B u_{t}, y-u_{t}\right\rangle+\varphi(y)-\varphi\left(u_{t}\right)+\frac{1}{r_{t}}\left\langle y-u_{t}, u_{t}-x_{t}\right\rangle \geqslant 0, \quad \forall y \in C, \\
x_{t}=(1-t)\left(\theta_{t} x_{t}+\left(1-\theta_{t}\right) T_{r_{t}} F_{r_{t}} K_{r_{t}} x_{t}\right), \quad \forall t \in(0,1) .
\end{array}\right.
$$

Then $\left\{x_{\mathrm{t}}\right\}$ and $\left\{\mathrm{u}_{\mathrm{t}}\right\}$ converge strongly, as $\mathrm{t} \rightarrow 0$, to a point $\mathrm{q} \in \Omega$, which solves the following minimum norm problem: find $x^{*} \in \Omega$ such that

$$
\left\|x^{*}\right\|=\min _{x \in \Omega}\|x\| \text {. }
$$

Proof. In (3.12) with $\mathrm{G}=\mathrm{I}, \mu=1, \tau=1, \mathrm{~V}=0$, and $\mathrm{l}=0$, letting $\mathrm{t} \rightarrow 0$ yields

$$
\|\mathrm{q}-\mathrm{p}\|^{2} \leqslant\langle p, p-q\rangle, \quad \forall p \in \Omega \text {. }
$$

Equivalently,

$$
\langle q, p-q\rangle \geqslant 0, \quad \forall p \in \Omega
$$

This obviously implies that

$$
\|\mathrm{q}\|^{2} \leqslant\langle\mathrm{p}, \mathrm{q}\rangle \leqslant\|\mathrm{p}\|\|\mathrm{q}\|, \quad \forall \mathrm{p} \in \Omega .
$$

It turns out that $\|q\| \leqslant\|p\|$ for all $p \in \Omega$. Therefore, $q$ is the minimum-norm point of $\Omega$.

Now, we propose the following modified hybrid iterative method which generates a sequence in an explicit way:

$$
\left\{\begin{array}{l}
\Theta\left(u_{n}, y\right)+\left\langle B u_{n}, y-u_{n}\right\rangle+\varphi(y)-\varphi\left(u_{n}\right)+\frac{1}{r_{n}}\left\langle y-u_{n}, u_{n}-x_{n}\right\rangle \geqslant 0, \quad \forall y \in C, \\
y_{n}=\beta_{n} x_{n}+\left(1-\beta_{n}\right) T_{r_{n}} F_{r_{n}} K_{r_{n}} x_{n}, \\
x_{n+1}=\alpha_{n} \gamma V x_{n}+\left(I-\alpha_{n} \mu G\right) y_{n}, \quad \forall n \geqslant 1,
\end{array}\right.
$$

where $\left\{\alpha_{n}\right\},\left\{\beta_{n}\right\} \subset(0,1) ;\left\{r_{n}\right\} \subset(0, \infty)$; and $x_{1} \in C$ is an arbitrary initial guess. 
Theorem 3.3. Let $\left\{x_{n}\right\}$ and $\left\{u_{n}\right\}$ be sequences generated by the explicit method (3.25). Let $\left\{\alpha_{n}\right\},\left\{\beta_{n}\right\}$ and $\left\{r_{n}\right\}$ satisfy the conditions:

(C1) $\lim _{n \rightarrow \infty} \alpha_{n}=0$;

(C2) $\sum_{n=1}^{\infty} \alpha_{n}=\infty$;

(C3) $0<\liminf _{n \rightarrow \infty} \beta_{n} \leqslant \limsup _{n \rightarrow \infty} \beta_{n}<1$;

(C4) $\lim _{n \rightarrow \infty}\left|r_{n+1}-r_{n}\right|=0$.

Then $\left\{x_{n}\right\}$ and $\left\{u_{n}\right\}$ converge strongly to $\mathrm{q} \in \Omega$, which is the unique solution of the variational inequality (3.2).

Proof. Note that from the condition (C1), without loss of generality, we assume that $\alpha_{n}(\tau-\gamma l)<1$ for $n \geqslant 1$. From now, we put $u_{n}=K_{r_{n}} x_{n}, z_{n}=F_{r_{n}} u_{n}$ and $w_{n}=T_{r_{n}} z_{n}$, for $n \geqslant 1$.

Now, we divide the proof into several steps.

Step 1. We show that $\left\{x_{n}\right\}$ is bounded. To this end, let $p \in \Omega$. Then, by Lemma 2.1 (iv), Lemma 2.2 (iii) and Lemma 2.3 (iii), $p=K_{r_{n}} p, p=F_{r_{n}} p$ and $p=T_{r_{n}} p$. From $z_{n}=F_{r_{n}} u_{n}$ and the fact that $F_{r_{n}}$ is nonexpansive, it follows that

$$
\left\|z_{n}-p\right\| \leqslant\left\|F_{r_{n}} u_{n}-F_{r_{n}} p\right\| \leqslant\left\|u_{n}-p\right\|
$$

Also, by $u_{n}=K_{r_{n}} x_{n}$,

$$
\left\|u_{n}-p\right\|=\left\|K_{r_{n}} x_{n}-K_{r_{n}} p\right\| \leqslant\left\|x_{n}-p\right\|
$$

and so

$$
\left\|z_{n}-p\right\| \leqslant\left\|x_{n}-p\right\|
$$

Now, by (3.26), we obtain that

$$
\begin{aligned}
\left\|y_{n}-p\right\| & =\left\|\beta_{n} x_{n}+\left(I-\beta_{n}\right) T_{r_{n}} z_{n}-p\right\| \\
& \leqslant \beta_{n}\left\|x_{n}-p\right\|+\left\|\left(I-\beta_{n}\right) T_{r_{n}} z_{n}-\left(I-\beta_{n}\right) T_{r_{n}} p\right\| \\
& \leqslant \beta_{n}\left\|x_{n}-p\right\|+\left(1-\beta_{n}\right)\left\|z_{n}-p\right\| \\
& \leqslant \beta_{n}\left\|x_{n}-p\right\|+\left(1-\beta_{n}\right)\left\|x_{n}-p\right\| \\
& =\left\|x_{n}-p\right\| .
\end{aligned}
$$

Thus, noting Lemma 2.8 and (3.27), we have

$$
\begin{aligned}
\left\|x_{n+1}-p\right\| & \leqslant \alpha_{n} \|\left(\gamma V\left(x_{n}\right)-\gamma V(p)\|+\|\left(I-\alpha_{n} \mu G\right) y_{n}-\left(I-\alpha_{n} \mu G\right) p\left\|+\alpha_{n}\right\| \gamma V(p)-\mu G p \|\right. \\
& \leqslant \alpha_{n} \gamma l\left\|x_{n}-p\right\|+\left(1-\alpha_{n} \tau\right)\left\|y_{n}-p\right\|+\alpha_{n}\|\gamma V(p)-\mu G p\| \\
& \leqslant \alpha_{n} \gamma l\left\|x_{n}-p\right\|+\left(1-\alpha_{n} \tau\right)\left\|x_{n}-p\right\|+\alpha_{n}\|\gamma V(p)-\mu G p\| \\
& =\left(1-(\tau-\gamma l) \alpha_{n}\right)\left\|x_{n}-p\right\|+\alpha_{n}\|\gamma V(p)-\mu G p\| .
\end{aligned}
$$

By induction, it follows from (3.28) that

$$
\left\|x_{n}-p\right\| \leqslant \max \left\{\left\|x_{1}-p\right\|, \frac{\|\gamma V(p)-\mu G p\|}{\tau-\gamma l}\right\}, \quad \forall n \geqslant 1
$$

Therefore $\left\{x_{n}\right\}$ is bounded, and so $\left\{u_{n}\right\},\left\{z_{n}\right\},\left\{y_{n}\right\},\left\{V\left(x_{n}\right)\right\},\left\{F u_{n}\right\},\left\{G y_{n}\right\}$, and $\left\{G_{r_{n}} z_{n}\right\}$ are bounded. Moreover, since $\left\|T_{n} z_{n}-p\right\| \leqslant\left\|x_{n}-p\right\|,\left\{T_{r_{n}} z_{n}\right\}$ is also bounded.

Step 2. We show that $\lim _{n \rightarrow \infty}\left\|x_{n+1}-x_{n}\right\|=0$ and $\lim _{n \rightarrow \infty}\left\|u_{n+1}-u_{n}\right\|=0$. Indeed, since $z_{n}=F_{r_{n}} u_{n}$, and $z_{n-1}=\mathrm{F}_{\mathrm{r}_{n-1}} \mathrm{u}_{\mathrm{n}-1}$, we get

$$
\left\langle y-z_{n}, F z_{n}\right\rangle+\frac{1}{r_{n}}\left\langle y-z_{n}, z_{n}-u_{n}\right\rangle \geqslant 0, \quad \forall y \in C,
$$


and

$$
\left\langle y-z_{n-1}, F z_{n-1}\right\rangle+\frac{1}{r_{n-1}}\left\langle y-z_{n-1}, z_{n-1}-u_{n-1}\right\rangle \geqslant 0, \quad \forall y \in C .
$$

Putting $y=z_{n-1}$ in (3.29) and $y=z_{\mathfrak{n}}$ in (3.30), we obtain

$$
\left\langle z_{n-1}-z_{n}, F z_{n}\right\rangle+\frac{1}{r_{n}}\left\langle z_{n-1}-z_{n}, z_{n}-u_{n}\right\rangle \geqslant 0,
$$

and

$$
\left\langle z_{\mathfrak{n}}-z_{\mathrm{n}-1}, F z_{\mathrm{n}-1}\right\rangle+\frac{1}{r_{\mathrm{n}-1}}\left\langle z_{\mathrm{n}}-z_{\mathrm{n}-1}, z_{\mathrm{n}-1}-\mathrm{u}_{\mathrm{n}-1}\right\rangle \geqslant 0 .
$$

Adding up (3.31) and (3.32), we derive

$$
-\left\langle z_{n}-z_{n-1}, F z_{n}-F z_{n-1}\right\rangle+\left\langle z_{n-1}-z_{n}, \frac{z_{n}-u_{n}}{r_{n}}-\frac{z_{n-1}-u_{n-1}}{r_{n-1}}\right\rangle \geqslant 0 .
$$

Since $\mathrm{F}$ is monotone, we have

$$
\left\langle z_{n-1}-z_{n}, \frac{z_{n}-u_{n}}{r_{n}}-\frac{z_{n-1}-u_{n-1}}{r_{n-1}}\right\rangle \geqslant 0,
$$

and hence

$$
\left\langle z_{n}-z_{n-1}, z_{n-1}-z_{n}+z_{n}-u_{n-1}-\frac{r_{n-1}}{r_{n}}\left(z_{n}-u_{n}\right)\right\rangle \geqslant 0 .
$$

Without loss of generality, let us assume that there exists a real number $r_{n}>b>0$ for $n \geqslant 1$. Then, from (3.33), we get

$$
\begin{aligned}
\left\|z_{n}-z_{n-1}\right\|^{2} & \leqslant\left\langle z_{n}-z_{n-1}, z_{n}-u_{n}+u_{n}-u_{n-1}-\frac{r_{n-1}}{r_{n}}\left(z_{n}-u_{n}\right)\right\rangle \\
& =\left\langle z_{n}-z_{n-1}, u_{n}-u_{n-1}+\left(1-\frac{r_{n-1}}{r_{n}}\right)\left(z_{n}-u_{n}\right)\right\rangle \\
& \leqslant\left\|z_{n}-z_{n-1}\right\|\left[\left\|u_{n}-u_{n-1}\right\|+\frac{1}{r_{n}}\left|r_{n}-r_{n-1}\right|\left\|z_{n}-u_{n}\right\|\right] .
\end{aligned}
$$

This implies that

$$
\left\|z_{n}-z_{n-1}\right\| \leqslant\left\|u_{n}-u_{n-1}\right\|+\frac{1}{b}\left|r_{n}-r_{n-1}\right| M_{1}
$$

where $M_{1}=\sup \left\{\left\|z_{n}-u_{n}\right\|: n \geqslant 1\right\}$.

On the other hand, from $u_{n-1}=K_{r_{n-1}} x_{n-1}$ and $u_{n}=K_{r_{n}} x_{n}$, it follows that

$$
\Theta\left(u_{n-1}, y\right)+\left\langle B u_{n-1}, y-u_{n-1}\right\rangle+\varphi(y)-\varphi\left(u_{n-1}\right)+\frac{1}{r_{n-1}}\left\langle y-u_{n-1}, u_{n-1}-x_{n-1}\right\rangle \geqslant 0, \quad \forall y \in C,
$$

and

$$
\Theta\left(u_{n}, y\right)+\left\langle B u_{n}, y-u_{n}\right\rangle+\varphi(y)-\varphi\left(u_{n}\right)+\frac{1}{r_{n}}\left\langle y-u_{n}, u_{n}-x_{n}\right\rangle \geqslant 0, \quad \forall y \in C .
$$

Substituting $y=u_{n}$ into (3.35) and $y=u_{n-1}$ into (3.36), we obtain

$$
\Theta\left(u_{n-1}, u_{n}\right)+\left\langle B u_{n-1}, u_{n}-u_{n-1}\right\rangle+\varphi\left(u_{n}\right)-\varphi\left(u_{n-1}\right)+\frac{1}{r_{n-1}}\left\langle u_{n}-u_{n-1}, u_{n-1}-x_{n-1}\right\rangle \geqslant 0,
$$

and

$$
\Theta\left(u_{n}, u_{n-1}\right)+\left\langle B u_{n}, u_{n-1}-u_{n}\right\rangle+\varphi\left(u_{n-1}\right)-\varphi\left(u_{n}\right)+\frac{1}{r_{n}}\left\langle u_{n-1}-u_{n}, u_{n}-x_{n}\right\rangle \geqslant 0 .
$$


By (A2), we have

$$
\left\langle u_{n}-u_{n-1}, B u_{n-1}-B u_{n}+\frac{u_{n-1}-x_{n-1}}{r_{n-1}}-\frac{u_{n}-x_{n}}{r_{n}}\right\rangle \geqslant 0,
$$

and then

$$
\left\langle u_{n}-u_{n-1}, r_{n-1}\left(B u_{n-1}-B u_{n}\right)+u_{n-1}-x_{n-1}-\frac{r_{n-1}}{r_{n}}\left(u_{n}-x_{n}\right)\right\rangle \geqslant 0 .
$$

So, it follows that

$$
\begin{aligned}
& \left\langle u_{n}-u_{n-1}, u_{n-1}-u_{n}\right\rangle+r_{n-1}\left\langle u_{n}-u_{n-1}, B u_{n-1}-B u_{n}\right\rangle \\
& \quad+\left\langle u_{n}-u_{n-1}, x_{n}-x_{n-1}\right\rangle+\left(1-\frac{r_{n-1}}{r_{n}}\right)\left\langle u_{n}-u_{n-1}, u_{n}-x_{n}\right\rangle \geqslant 0 .
\end{aligned}
$$

Then, from (3.37), $r_{n}>b>0$ for $n \geqslant 1$, and the fact that $\left\langle u_{n}-u_{n-1}, B u_{n-1}-B u_{n}\right\rangle \leqslant 0$, we have

$$
\begin{aligned}
\left\|u_{n}-u_{n-1}\right\|^{2} & \leqslant\left\langle u_{n}-u_{n-1}, x_{n}-x_{n-1}\right\rangle+\left(1-\frac{r_{n-1}}{r_{n}}\right)\left\langle u_{n}-u_{n-1}, u_{n}-x_{n}\right\rangle \\
& \leqslant\left\|u_{n}-u_{n-1}\right\|\left[\left\|x_{n}-x_{n-1}\right\|+\left|1-\frac{r_{n-1}}{r_{n}}\right|\left\|u_{n}-x_{n}\right\|\right],
\end{aligned}
$$

which implies that

$$
\begin{aligned}
\left\|u_{n}-u_{n-1}\right\| & \leqslant\left\|x_{n}-x_{n-1}\right\|+\frac{1}{r_{n}}\left|r_{n}-r_{n-1}\right|\left\|u_{n}-x_{n}\right\| \\
& \leqslant\left\|x_{n}-x_{n-1}\right\|+\frac{1}{b}\left|r_{n}-r_{n-1}\right| M_{2},
\end{aligned}
$$

where $M_{2}=\sup \left\{\left\|u_{n}-x_{n}\right\|: n \geqslant 1\right\}$. Substituting (3.38) into (3.34), we have

$$
\left\|z_{n}-z_{n-1}\right\| \leqslant\left\|x_{n}-x_{n-1}\right\|+\frac{1}{b}\left|r_{n}-r_{n-1}\right|\left(M_{1}+M_{2}\right) \text {. }
$$

On another hand, let $w_{n}=\mathrm{T}_{\mathrm{r}_{n}} z_{n}$ and $w_{n-1}=\mathrm{T}_{\mathrm{r}_{n-1}} z_{n-1}$. Then we get

$$
\left\langle y-w_{n-1}, T w_{n-1}\right\rangle-\frac{1}{r_{n-1}}\left\langle y-w_{n-1},\left(1+r_{n-1}\right) w_{n-1}-z_{n-1}\right\rangle \leqslant 0, \quad \forall y \in C,
$$

and

$$
\left\langle y-w_{n}, T w_{n}\right\rangle-\frac{1}{r_{n}}\left\langle y-w_{n},\left(1+r_{n}\right) w_{n}-z_{n}\right\rangle \leqslant 0, \quad \forall y \in C .
$$

Putting $y=w_{n}$ in (3.40) and $y=w_{n-1}$ in (3.41), we obtain

$$
\left\langle w_{n}-w_{n-1}, T w_{n-1}\right\rangle-\frac{1}{r_{n-1}}\left\langle w_{n}-w_{n-1},\left(1+r_{n-1}\right) w_{n-1}-z_{n-1}\right\rangle \leqslant 0,
$$

and

$$
\left\langle w_{n-1}-w_{n}, T w_{n}\right\rangle-\frac{1}{r_{n}}\left\langle w_{n-1}-w_{n},\left(1+r_{n}\right) w_{n}-z_{n}\right\rangle \leqslant 0 .
$$

Adding up (3.42) and (3.43), we have

$$
\left\langle w_{n}-w_{n-1}, T w_{n-1}-T w_{n}\right\rangle-\left\langle w_{n}-w_{n-1}, \frac{\left(1+r_{n-1}\right) w_{n-1}-z_{n-1}}{r_{n-1}}-\frac{\left(1+r_{n}\right) w_{n}-z_{n}}{r_{n}}\right\rangle \leqslant 0,
$$

which implies that

$$
\left\langle w_{n}-w_{n-1},\left(w_{n}-T w_{n}\right)-\left(w_{n-1}-T w_{n-1}\right)\right\rangle-\left\langle w_{n}-w_{n-1}, \frac{w_{n-1}-z_{n-1}}{r_{n-1}}-\frac{w_{n}-z_{n}}{r_{n}}\right\rangle \leqslant 0 .
$$

Now, using the fact that $\mathrm{T}$ is pseudocontractive, we induce

$$
\left\langle w_{n}-w_{n-1}, \frac{w_{n-1}-z_{n-1}}{r_{n-1}}-\frac{w_{n}-z_{n}}{r_{n}}\right\rangle \geqslant 0,
$$

and hence 


$$
\left\langle w_{n}-w_{n-1}, w_{n-1}-w_{n}+w_{n}-z_{n-1}-\frac{r_{n-1}}{r_{n}}\left(w_{n}-z_{n}\right)\right\rangle \geqslant 0 .
$$

Since $r_{n}>b>0$ for $n \geqslant 1$, by (3.44), we have

$$
\begin{aligned}
\left\|w_{n}-w_{n-1}\right\|^{2} & \leqslant\left\langle w_{n}-w_{n-1}, z_{n}-z_{n-1}+\left(1-\frac{r_{n-1}}{r_{n}}\right)\left(w_{n}-z_{n}\right)\right\rangle \\
& \leqslant\left\|w_{n}-w_{n-1}\right\|\left[\left\|z_{n}-z_{n-1}\right\|+\frac{1}{r_{n}}\left|r_{n}-r_{n-1}\right|\left\|w_{n}-z_{n}\right\|\right],
\end{aligned}
$$

which implies

$$
\left\|w_{n}-w_{n-1}\right\| \leqslant\left\|z_{n}-z_{n-1}\right\|+\frac{1}{b}\left|r_{n}-r_{n-1}\right| M_{3},
$$

where $M_{3}=\sup \left\{\left\|w_{n}-z_{n}\right\|: n \geqslant 1\right\}$. From (3.39) and (3.45), it follows that

$$
\left\|\mathrm{T}_{\mathrm{r}_{n}} z_{\mathrm{n}}-\mathrm{T}_{\mathrm{r}_{\mathrm{n}-1}} z_{\mathrm{n}-1}\right\| \leqslant\left\|\mathrm{x}_{\mathrm{n}}-\mathrm{x}_{\mathrm{n}-1}\right\|+\frac{1}{\mathrm{~b}}\left|\mathrm{r}_{\mathrm{n}}-\mathrm{r}_{\mathrm{n}-1}\right|\left(\mathrm{M}_{1}+\mathrm{M}_{2}+\mathrm{M}_{3}\right) .
$$

Now, define

$$
x_{n+1}=\beta_{n} x_{n}+\left(1-\beta_{n}\right) k_{n}, \quad \forall n \geqslant 1 .
$$

Then, from the definition of $k_{n}$, we obtain

$$
\begin{aligned}
k_{n+1}-k_{n}= & \frac{x_{n+2}-\beta_{n+1} x_{n+1}}{1-\beta_{n+1}}-\frac{x_{n+1}-\beta_{n} x_{n}}{1-\beta_{n}} \\
= & \frac{\alpha_{n+1} \gamma V\left(x_{n+1}\right)+\left(I-\alpha_{n+1} \mu G\right) y_{n+1}-\beta_{n+1} x_{n+1}}{1-\beta_{n+1}}-\frac{\alpha_{n} \gamma V\left(x_{n}\right)+\left(I-\alpha_{n} G\right) y_{n}-\beta_{n} x_{n}}{1-\beta_{n}} \\
= & \frac{\alpha_{n+1} \gamma V\left(x_{n+1}\right)}{1-\beta_{n+1}}-\frac{\alpha_{n} \gamma V\left(x_{n}\right)}{1-\beta_{n}}-\frac{\left(I-\alpha_{n} \mu G\right)\left(\beta_{n} x_{n}+\left(1-\beta_{n}\right) T_{r_{n}} z_{n}\right)-\beta_{n} x_{n}}{1-\beta_{n} x_{n}} \\
& +\frac{\left(I-\alpha_{n+1} \mu G\right)\left(\beta_{n+1} x_{n+1}+\left(1-\beta_{n+1}\right) T_{r_{n+1}} z_{n+1}\right)-\beta_{n+1} x_{n+1}}{1-\beta_{n+1} x_{n+1}} \\
= & \frac{\alpha_{n+1} \gamma V\left(x_{n+1}\right)}{1-\beta_{n+1}}-\frac{\alpha_{n} \gamma V\left(x_{n}\right)}{1-\beta_{n}}-\frac{\beta_{n} x_{n}+\left(1-\beta_{n}\right) T_{r_{n}} z_{n}-\beta_{n} x_{n}}{1-\beta_{n}}+\frac{\alpha_{n} \mu G y_{n}}{1-\beta_{n}} \\
& +\frac{\beta_{n+1} x_{n+1}+\left(1-\beta_{n+1}\right) T_{r_{n+1}} x_{n+1}-\beta_{n+1} x_{n+1}}{1-\beta_{n+1}}-\frac{\left.\alpha_{n+1} \mu G\right) y_{n+1}}{1-\beta_{n+1}} \\
= & \frac{\alpha_{n+1}}{1-\beta_{n+1}}\left(\gamma V\left(x_{n+1}\right)-\mu G y_{n+1}\right)-\frac{\alpha_{n}}{1-\beta_{n}}\left(\gamma V\left(x_{n}\right)-\mu G y_{n}\right)+T_{r_{n+1}} z_{n+1}-T_{r_{n}} z_{n} \\
= & \frac{\alpha_{n+1}}{1-\beta_{n+1}}\left(\gamma V\left(x_{n+1}\right)-\mu G y_{n+1}\right)-\frac{\alpha_{n}}{1-\beta_{n}}\left(\gamma V\left(x_{n}\right)-\mu G y_{n}\right) \\
& +T_{r_{n+1}} z_{n+1}-T_{r_{n+1}} z_{n}+\left(T_{r_{n+1}} z_{n}-T_{r_{n}} z_{n}\right) .
\end{aligned}
$$

So, it follows from (3.46) that

$$
\begin{aligned}
\left\|k_{n+1}-k_{n}\right\|- & \left\|x_{n+1}-x_{n}\right\| \\
\leqslant & \frac{\alpha_{n+1}}{1-\beta_{n+1}}\left(\gamma\left\|V\left(x_{n+1}\right)\right\|+\mu\left\|G y_{n+1}\right\|\right)+\frac{\alpha_{n}}{1-\beta_{n}}\left(\gamma\left\|V\left(x_{n}\right)\right\|+\mu\left\|G y_{n}\right\|\right) \\
& +\left\|T_{n+1} z_{n+1}-T_{n+1} z_{n}\right\|+\left\|T_{r_{n+1}} z_{n}-T_{r_{n}} z_{n}\right\| \\
\leqslant & \frac{\alpha_{n+1}}{1-\beta_{n+1}}\left(\gamma\left\|V\left(x_{n+1}\right)\right\|+\mu\left\|G y_{n+1}\right\|\right)+\frac{\alpha_{n}}{1-\beta_{n}}\left(\gamma\left\|V\left(x_{n}\right)\right\|+\mu\left\|G y_{n}\right\|\right) \\
& \quad+\left\|z_{n+1}-z_{n}\right\|+\frac{1}{b}\left|r_{n}-r_{n-1}\right| M_{3} \\
\leqslant & \left(\frac{\alpha_{n+1}}{1-\beta_{n+1}}+\frac{\alpha_{n}}{1-\beta_{n}}\right) M_{4}+\left\|z_{n+1}-z_{n}\right\|+\frac{1}{b}\left|r_{n}-r_{n-1}\right| M_{3} \\
\leqslant & \left(\frac{\alpha_{n+1}}{1-\beta_{n+1}}+\frac{\alpha_{n}}{1-\beta_{n}}\right) M_{4}+\left\|x_{n}-x_{n-1}\right\|+\frac{1}{b}\left|r_{n}-r_{n-1}\right|\left(M_{1}+M_{2}+M_{3}\right),
\end{aligned}
$$


where $M_{4}=\sup \left\{\gamma\left\|V\left(x_{n}\right)\right\|+\mu\left\|G y_{n}\right\|: n \geqslant 1\right\}$. Thus, by conditions (C1), (C3) and (C4), from (3.47) we have

$$
\limsup _{n \rightarrow \infty}\left(\left\|k_{n+1}-k_{n}\right\|-\left\|x_{n+1}-x_{n}\right\|\right) \leqslant 0
$$

Hence, by Lemma 2.5,

$$
\lim _{n \rightarrow \infty}\left\|k_{n}-x_{n}\right\|=0
$$

Consequently,

$$
\lim _{n \rightarrow \infty}\left\|x_{n+1}-x_{n}\right\|=\lim _{n \rightarrow \infty}\left(1-\beta_{n}\right)\left\|k_{n}-x_{n}\right\|=0 .
$$

Also from (3.38) and (3.39), it follows that

$$
\lim _{n+o \infty}\left\|u_{n+1}-u_{n}\right\|=0 \text { and } \lim _{n \rightarrow \infty}\left\|z_{n+1}-z_{n}\right\|=0 .
$$

Step 3. We show that $\lim _{n \rightarrow \infty}\left\|x_{n}-w_{n}\right\|=\lim _{n \rightarrow \infty}\left\|x_{n}-T_{r_{n}} z_{n}\right\|=0$. Noting that $x_{n+1}=\alpha_{n} \gamma V\left(x_{n}\right)+$ $\left(I-\alpha_{n} \mu G\right) y_{n}$, we have

$$
\begin{aligned}
\left\|x_{n}-T_{r_{n}} z_{n}\right\| & \leqslant\left\|x_{n}-x_{n+1}\right\|+\left\|x_{n+1}-T_{r_{n}} z_{n}\right\| \\
& \leqslant\left\|x_{n}-x_{n+1}\right\|+\alpha_{n}\left\|\gamma V\left(x_{n}\right)-\mu G y_{n}\right\|+\left\|y_{n}-T_{r_{n}} z_{n}\right\| \\
& =\left\|x_{n}-x_{n+1}\right\|+\alpha_{n}\left\|\gamma V\left(x_{n}\right)-\mu G y_{n}\right\|+\left\|\beta_{n} x_{n}+\left(1-\beta_{n}\right) T_{r_{n}} z_{n}-T_{r_{n}} z_{n}\right\| \\
& =\left\|x_{n}-x_{n+1}\right\|+\alpha_{n}\left\|\gamma V\left(x_{n}\right)-\mu G y_{n}\right\|+\beta_{n}\left\|x_{n}-T_{r_{n}} z_{n}\right\|,
\end{aligned}
$$

that is,

$$
\left\|x_{n}-T_{r_{n}} z_{n}\right\| \leqslant \frac{1}{1-\beta_{n}}\left\|x_{n}-x_{n+1}\right\|+\frac{\alpha_{n}}{1-\beta_{n}}\left\|\gamma V\left(x_{n}\right)-\mu G y_{n}\right\|
$$

From the conditions (C1), (C3) and Step 2, it follows that

$$
\lim _{n \rightarrow \infty}\left\|x_{n}-w_{n}\right\|=\lim _{n \rightarrow \infty}\left\|x_{n}-T_{r_{n}} z_{n}\right\|=0 .
$$

Step 4. We show that $\lim _{n \rightarrow \infty}\left\|x_{n}-u_{n}\right\|=0$. To this end, let $p \in \Omega$. Since $K_{r_{n}}$ is firmly nonexpansive and $u_{n}=K_{r_{n}} x_{n}$, we have

$$
\begin{aligned}
\left\|u_{n}-p\right\|^{2} & =\left\|K_{r_{n}} x_{n}-K_{r_{n}} p\right\|^{2} \\
& \leqslant\left\langle K_{r_{n}} x_{n}-K_{r_{n}} p, x_{n}-x_{n}-p\right\rangle \\
& =\frac{1}{2}\left(\left\|u_{n}-p\right\|^{2}+\left\|x_{n}-p\right\|^{2}\right)-\frac{1}{2}\left(\left\|x_{n}-p-\left(u_{n}-p\right)\right\|^{2}\right) \\
& =\frac{1}{2}\left(\left\|u_{n}-p\right\|^{2}+\left\|x_{n}-p\right\|^{2}-\left\|x_{n}-u_{n}\right\|^{2}\right)
\end{aligned}
$$

and hence

$$
\left\|u_{n}-p\right\|^{2} \leqslant\left\|x_{n}-p\right\|^{2}-\left\|x_{n}-u_{n}\right\|^{2}
$$

From $z_{n}=F_{r_{n}} u_{n}$, we also get

$$
\left\|z_{n}-p\right\|^{2}=\left\|F_{r_{n}} u_{n}-F_{r_{n}} p\right\|^{2} \leqslant\left\|u_{n}-p\right\|^{2} .
$$

By (3.48) and (3.49), we obtain

$$
\begin{aligned}
\left\|x_{n+1}-p\right\|^{2}= & \left\|\alpha_{n}\left(\gamma V\left(x_{n}\right)-\mu G y_{n}\right)+\left(y_{n}-p\right)\right\|^{2} \\
= & \left\|\alpha_{n}\left(\gamma V\left(x_{n}\right)-\mu G y_{n}\right)+\beta_{n}\left(x_{n}-T_{r_{n}} z_{n}\right)+\left(T_{r_{n}} z_{n}-p\right)\right\|^{2} \\
\leqslant & {\left[\left(\left\|\alpha_{n}\left(\gamma V\left(x_{n}\right)-\mu G y_{n}\right)\right\|+\left\|z_{n}-p\right\|\right)+\beta_{n}\left\|x_{n}-T_{r_{n}} z_{n}\right\|\right]^{2} } \\
= & \alpha_{n}^{2}\left\|\gamma V\left(x_{n}\right)-\mu G y_{n}\right\|^{2}+2 \alpha_{n}\left\|\gamma V\left(x_{n}\right)-\mu G y_{n}\right\|\left\|z_{n}-p\right\|+\left\|z_{n}-p\right\|^{2} \\
& +\beta_{n}\left\|x_{n}-T_{n} z_{n}\right\|\left[2\left(\alpha_{n}\left\|\gamma V\left(x_{n}\right)-\mu G y_{n}\right\|+\left\|z_{n}-p\right\|\right)+\beta_{n}\left\|x_{n}-T_{r_{n}} z_{n}\right\|\right] \\
\leqslant & \alpha_{n}\left\|\gamma V\left(x_{n}\right)-\mu G y_{n}\right\|^{2}+\left\|u_{n}-p\right\|^{2}+M_{n} \\
\leqslant & \alpha_{n}\left\|\gamma V\left(x_{n}\right)-\mu G y_{n}\right\|^{2}+\left(\left\|x_{n}-p\right\|^{2}-\left\|x_{n}-u_{n}\right\|^{2}\right)+M_{n},
\end{aligned}
$$


where

$$
\begin{aligned}
M_{n}= & \beta_{n}\left\|x_{n}-w_{n}\right\|\left[2\left(\alpha_{n}\left\|\gamma V\left(x_{n}\right)-\mu G y_{n}\right\|+\left\|z_{n}-p\right\|\right)+\beta_{n}\left\|x_{n}-w_{n}\right\|\right] \\
& +2 \alpha_{n}\left\|\gamma V\left(x_{n}\right)-\mu G y_{n}\right\|\left\|z_{n}-p\right\| .
\end{aligned}
$$

Now, from (3.50), we derive

$$
\begin{aligned}
\left\|x_{n}-u_{n}\right\|^{2} & \leqslant \alpha_{n}\left\|\gamma V\left(x_{n}\right)-\mu G y_{n}\right\|^{2}+M_{n}+\left\|x_{n}-p\right\|^{2}-\left\|x_{n+1}-p\right\|^{2} \\
& \leqslant \alpha_{n}\left\|\gamma V\left(x_{n}\right)-\mu G y_{n}\right\|^{2}+M_{n}+\left\|x_{n}-x_{n+1}\right\|\left(\left\|x_{n}-p\right\|+\left\|x_{n+1}-p\right\|\right) .
\end{aligned}
$$

Since $\alpha_{n} \rightarrow 0$ by the condition (C1), $\left\|x_{n+1}-x_{n}\right\| \rightarrow 0$ by Step 2 and $M_{n} \rightarrow 0$ by Step 3 and the condition (C1), we have

$$
\lim _{n \rightarrow \infty}\left\|x_{n}-u_{n}\right\|=0 .
$$

Step 5. We show that $\lim _{n \rightarrow \infty}\left\|u_{n}-z_{n}\right\|=0$. To this end, let $p \in \Omega$. Using $z_{n}=F_{r_{n}} u_{n}$ and $p=F_{r_{n}} p$, and firmly nonexpansivity of $F_{r_{n}}$, we observe that

$$
\begin{aligned}
\left\|z_{n}-p\right\|^{2} & =\left\|F_{r_{n}} u_{n}-F_{r_{n}} p\right\|^{2} \\
& \leqslant\left\langle F_{r_{n}} u_{n}-F_{r_{n}} p, u_{n}-p\right\rangle \\
& =\left\langle z_{n}-p, u_{n}-p\right\rangle \\
& \leqslant \frac{1}{2}\left(\left\|z_{n}-p\right\|^{2}+\left\|u_{n}-p\right\|^{2}-\left\|u_{n}-z_{n}\right\|^{2}\right),
\end{aligned}
$$

that is,

$$
\left\|z_{n}-p\right\|^{2} \leqslant\left\|u_{n}-p\right\|^{2}-\left\|u_{n}-z_{n}\right\|^{2} \leqslant\left\|x_{n}-p\right\|^{2}-\left\|u_{n}-z_{n}\right\|^{2} .
$$

Now, from (3.50) and (3.52), we compute

$$
\begin{aligned}
\left\|x_{n+1}-p\right\|^{2} & \leqslant \alpha_{n}\left\|\gamma V\left(x_{n}\right)-\mu G y_{n}\right\|^{2}+\left\|z_{n}-p\right\|^{2}+M_{n} \\
& \leqslant \alpha_{n}\left\|\gamma V\left(x_{n}\right)-\mu G y_{n}\right\|^{2}+\left\|x_{n}-p\right\|^{2}-\left\|u_{n}-z_{n}\right\|^{2}+M_{n},
\end{aligned}
$$

where $M_{n}$ is of in (3.51). So, we get

$$
\begin{aligned}
\left\|u_{n}-z_{n}\right\|^{2} & \leqslant \alpha_{n}\left\|\gamma V\left(x_{n}\right)-\mu G y_{n}\right\|^{2}+M_{n}+\left\|x_{n}-p\right\|^{2}-\left\|x_{n+1}-p\right\|^{2} \\
& \leqslant \alpha_{n}\left\|\gamma V\left(x_{n}\right)-\mu G y_{n}\right\|^{2}+M_{n}+\left\|x_{n+1}-x_{n}\right\|\left(\left\|x_{n}-p\right\|+\left\|x_{n+1}-p\right\|\right) .
\end{aligned}
$$

From the condition (C1), Step 2 and $\lim _{n \rightarrow \infty} M_{n}=0$, it follows that

$$
\lim _{n \rightarrow \infty}\left\|u_{n}-z_{n}\right\|=0 \text {. }
$$

Step 6. We show that $\lim _{n \rightarrow \infty}\left\|z_{n}-w_{n}\right\|=\lim _{n \rightarrow \infty}\left\|z_{n}-T_{r_{n}} z_{n}\right\|=0$. Since $\left\|w_{n}-z_{n}\right\| \leqslant\left\|w_{n}-x_{n}\right\|+$ $\left\|x_{n}-u_{n}\right\|+\left\|u_{n}-z_{n}\right\|$, by Step 3, Step 4 and Step 5, we conclude that

$$
\lim _{n \rightarrow \infty}\left\|w_{n}-z_{n}\right\|=\lim _{n \rightarrow \infty}\left\|T_{n} z_{n}-z_{n}\right\|=0 .
$$

Step 7. We show that $\lim _{n \rightarrow \infty}\left\|x_{n}-z_{n}\right\|=0$. Indeed, from Step 3 and Step 6, we have

$$
\left\|x_{n}-z_{n}\right\| \leqslant\left\|x_{n}-w_{n}\right\|+\left\|w_{n}-z_{n}\right\| \rightarrow 0 \text { as } n \rightarrow \infty .
$$

Step 8. We show that $\limsup _{n \rightarrow \infty}\left\langle(\gamma V-\mu G) q, x_{n}-q\right\rangle \leqslant 0$, where $q$ is a solution of the variational inequality (3.2). To this end, first we prove that

$$
\limsup _{n \rightarrow \infty}\left\langle(\gamma V-\mu G) q, w_{n}-q\right\rangle=\limsup _{n \rightarrow \infty}\left\langle(\gamma V-\mu G) q, T_{r_{n}} z_{n}-q\right\rangle \leqslant 0
$$


Since $\left\{z_{n}\right\}$ is bounded, we can choose a subsequence $\left\{z_{n_{i}}\right\}$ of $\left\{z_{n}\right\}$ such that

$$
\limsup _{n \rightarrow \infty}\left\langle(\gamma V-\mu \mathrm{G}) \mathbf{q}, w_{n}-\mathbf{q}\right\rangle=\lim _{i \rightarrow \infty}\left\langle(\gamma V-\mu \mathrm{G}) \mathbf{q}, w_{n_{i}}-q\right) .
$$

Without loss of generality, we may assume that $\left\{z_{n_{i}}\right\}$ converges weakly to $z \in C$. From $\left\|w_{n}-z_{n}\right\| \rightarrow 0$ by Step 6, it follows that $w_{n_{i}} \rightarrow z$. Moreover, from Step 3 and Step 4, it follows that $x_{n_{i}} \rightarrow z$ and $u_{n_{i}} \rightarrow z$. Thus, by the same argument as in Step 7 of the proof of Theorem 3.1 together with Step 4, Step 5 and Step 6 , we obtain $z \in \Omega$. So, from (3.53), we obtain

$$
\begin{aligned}
\limsup _{n \rightarrow \infty}\left\langle(\gamma V-\mu G) q, w_{n}-q\right. & =\lim _{i \rightarrow \infty}\left\langle(\gamma V-\mu G) q, w_{n_{i}}-q\right\rangle \\
& =\langle(\gamma V-\mu G) q, z-q\rangle \leqslant 0 .
\end{aligned}
$$

Since $\lim _{n \rightarrow \infty}\left\|x_{n}-w_{n}\right\|=0$ by Step 3, from (3.54), we conclude that

$$
\begin{aligned}
\limsup _{n \rightarrow \infty}\left\langle(\gamma V-\mu G) q, x_{n}-q\right\rangle \\
\quad \leqslant \limsup _{n \rightarrow \infty}\left\langle(\gamma V-\mu G) q, x_{n}-w_{n}\right\rangle+\limsup _{n \rightarrow \infty}\left\langle(\gamma V-\mu G) q, w_{n}-q\right\rangle \\
\quad \leqslant \limsup _{n \rightarrow \infty}\|(\gamma V-\mu G) q\|\left\|x_{n}-w_{n}\right\|+\limsup _{n \rightarrow \infty}\left\langle(\gamma V-\mu G) q, w_{n}-q\right) \leqslant 0 .
\end{aligned}
$$

Step 9. We show that $\lim _{n \rightarrow \infty}\left\|x_{n}-q\right\|=0$ and $\lim _{n \rightarrow \infty}\left\|u_{n}-q\right\|=0$, where $q$ is a solution of the variational inequality (3.2). Indeed, from (3.3), Lemma 2.5 and Lemma 2.8, we have

$$
\begin{aligned}
\left\|x_{n+1}-q\right\|^{2}= & \left\|\alpha_{n} \gamma V\left(x_{n}\right)+\left(I-\alpha_{n} \mu G\right) y_{n}-p\right\|^{2} \\
= & \left\|\alpha_{n}\left(\gamma V\left(x_{n}\right)-\gamma V(q)\right)+\left(I-\alpha_{n} \mu G\right) y_{n}-\left(I-\alpha_{n} \mu G\right) q+\alpha_{n}(\gamma V(q)-\mu G q)\right\|^{2} \\
\leqslant & {\left[\alpha_{n} \gamma l\left\|x_{n}-q\right\|+\left(1-\alpha_{n} \tau\right)\left\|y_{n}-q\right\|\right]^{2}+2 \alpha_{n}\left\langle(\gamma V-\mu G) q, x_{n+1}-q\right\rangle } \\
\leqslant & {\left[\alpha_{n} \gamma l\left\|x_{n}-q\right\|+\left(1-\alpha_{n} \tau\right)\left(\beta_{n}\left\|x_{n}-q\right\|+\left(1-\beta_{n}\right)\left\|T_{r_{n}} z_{n}-q\right\|\right)\right]^{2} } \\
& +2 \alpha_{n}\left\langle(\gamma V-\mu G) q, x_{n+1}-q\right\rangle \\
\leqslant & {\left[\alpha_{n} \gamma l\left\|x_{n}-q\right\|+\left(1-\alpha_{n} \tau\right)\left(\beta_{n}\left\|x_{n}-q\right\|+\left(1-\beta_{n}\right)\left\|z_{n}-q\right\|\right)\right]^{2} } \\
& +2 \alpha_{n}\left\langle(\gamma V-\mu G) q, x_{n+1}-q\right\rangle \\
\leqslant & {\left[\alpha_{n} \gamma l\left\|x_{n}-q\right\|+\left(1-\alpha_{n} \tau\right)\left\|x_{n}-q\right\|\right]^{2}+2 \alpha_{n}\left\langle(\gamma V-\mu G) q, x_{n+1}-q\right\rangle } \\
\leqslant & \left(1-(\tau-\gamma l) \alpha_{n}\right)\left\|x_{n}-q\right\|^{2}+2 \alpha_{n}\left\langle(\gamma V-\mu G) q, x_{n+1}-q\right\rangle \\
= & \left(1-\overline{\alpha_{n}}\right)\left\|x_{n}-q\right\|^{2}+\overline{\beta_{n}},
\end{aligned}
$$

where $\overline{\alpha_{n}}=(\tau-\gamma l) \alpha_{n}$ and $\overline{\beta_{n}}=2(\tau-\gamma l) \alpha_{n}\left\langle(\gamma V-\mu G) q, x_{n+1}-q\right\rangle$. From the conditions (C1) and (C2), and Step 8, it is easily seen that $\bar{\alpha}_{n} \rightarrow 0, \sum_{n=1}^{\infty} \bar{\alpha}_{n}=\infty$, and $\lim \sup _{n \rightarrow \infty} \frac{\bar{\beta}_{n}}{\bar{\alpha}_{n}} \leqslant 0$. Hence, by applying Lemma 2.4 to (3.55), we conclude $x_{n} \rightarrow q$ as $n \rightarrow \infty$. Moreover, by Step 4 , we obtain that $u_{n} \rightarrow q$ as $\mathrm{n} \rightarrow \infty$. This completes the proof.

From Theorem 3.3, we deduce immediately the following result.

Corollary 3.4. Let $\left\{x_{n}\right\}$ and $\left\{u_{n}\right\}$ be sequences generated by

$$
\left\{\begin{array}{l}
\Theta\left(u_{n}, y\right)+\left\langle B u_{n}, y-u_{n}\right\rangle+\varphi(y)-\varphi\left(u_{n}\right)+\frac{1}{r_{n}}\left\langle y-u_{n}, u_{n}-x_{n}\right\rangle \geqslant 0, \forall y \in C, \\
y_{n}=\beta_{n} x_{n}+\left(1-\beta_{n}\right) T_{r_{n}} F_{r_{n}} K_{r_{n}} x_{n}, \\
x_{n+1}=\left(1-\alpha_{n}\right) y_{n}, \quad \forall n \geqslant 1 .
\end{array}\right.
$$

Let $\left\{\alpha_{n}\right\},\left\{\beta_{n}\right\}$, and $\left\{r_{n}\right\}$ be sequences satisfying conditions (C1), (C2), (C3), and (C4) in Theorem 3.3. Then $\left\{x_{n}\right\}$ and $\left\{\mathrm{u}_{\mathrm{n}}\right\}$ converge strongly to a point $\mathrm{q} \in \Omega$, which solves the minimum norm problem (3.24). 
Proof. Take $\mathrm{G}=\mathrm{I}, \mu=1 \tau=1, \mathrm{~V}=0$, and $\mathrm{l}=0$ in Theorem 3.3. Then the variational inequality (3.2) is reduced to the inequality

$$
\langle q, p-q\rangle \geqslant 0, \quad \forall p \in \Omega .
$$

This is equivalent to $\|q\|^{2} \leqslant\langle p, q\rangle \leqslant\|p\|\|q\|$ for all $p \in \Omega$. It turns out that $\|q\| \leqslant\|p\|$ for all $p \in \Omega$ and $q$ is the minimum-norm point of $\Omega$.

\section{Remark 3.5.}

(1) For finding a common element of $\operatorname{GMEP}(\Theta, \varphi, B) \cap \operatorname{VI}(C, F) \cap \operatorname{Fix}(T)$, where $B$ is a continuous monotone mapping, $\mathrm{F}$ is a continuous monotone mapping, and $\mathrm{T}$ is a continuous pseudocontractive mapping, Theorem 3.1 and Theorem 3.3 are new ones different from previous those introduced by several authors. Consequently, in the sense that our convergence is for the more general class of continuous monotone mappings and the more general class of continuous pseudocontractive mappings, our results improve, develop and complement the corresponding results, which were obtained recently by several authors in references; for example, see $[5,6,8,12,13,15,16,19,24,25$, 27-29, 32, 36-39] and the references therein.

(2) We point out that Corollary 3.2 and Corollary 3.4 for finding the minimum-norm point of $\operatorname{GMEP}(\Theta, \varphi, \mathrm{B}) \cap \operatorname{VI}(\mathrm{C}, \mathrm{F}) \cap \operatorname{Fix}(\mathrm{T})$ are also new ones different from previous those introduced by several authors.

(3) We recall some special cases of the generalized mixed equilibrium problem (1.1) as follows:

(i) If $\Theta(x, y)=0$ for all $x, y \in C$, the GMEP (1.1) reduces the following generalized variational inequality problem (for short, GVI) of finding $x \in C$ such that

$$
\langle\mathrm{B} x, y-x\rangle+\varphi(y)-\varphi(x) \geqslant 0, \quad \forall y \in C .
$$

(ii) If $\mathrm{B}=0$ and $\Theta(x, y)=0$ for all $x, y \in C$, the GMEP (1.1) reduces the following minimization problem (for short, MP) finding $x \in C$ such that

$$
\varphi(y)-\varphi(x) \geqslant 0, \quad \forall y \in C .
$$

Applying Theorem 3.1, Theorem 3.3, Corollary 3.2, and Corollary 3.4, we can also establish the new corresponding results for the GEP (1.2), the MEP (1.3), the EP (1.4), the GVI (3.56) and the MP (3.57).

\section{Acknowledgment}

This study was supported by research funds from Dong-A University.

The author would like to thank the anonymous reviewers for their valuable suggestions and comments along with providing some recent related papers.

\section{References}

[1] R. P. Agarwal, D. O'Regan, D. R. Sahu, Fixed point theory for Lipschitzian-type mappings with applications, Topological Fixed Point Theory and Its Applications, Springer, New York, (2009). 2

[2] E. Blum, W. Oettli, From optimization and variational inequalities to equilibrium problems, Math. Student, 63 (1994), 123-145. 1

[3] L.-C. Ceng, S. Al-Homidan, Q. H. Ansari, J.-C. Yao, An iterative scheme for equilibrium problems and fixed point problems of strict pseudo-contraction mappings, J. Comput. Appl. Math., 223 (2009), 967-974. 1

[4] L.-C. Ceng, S.-M. Guu, J.-C. Yao, Hybrid iterative method for finding common solutions of generalized mixed equilibrium and fixed point problems, Fixed Point Theory Appl., 2012 (2012), 19 pages. 1

[5] L.-C. Ceng, J.-C. Yao, A hybrid iterative scheme for mixed equilibrium problems and fixed point problems, J. Comput. Appl. Math., 214 (2008), 186-201. 1, 1, 3.5

[6] S.-S. Chang, H. W. Joseph Lee, C. K. Chan, A new method for solving equilibrium problem fixed point problem and variational inequality problem with application to optimization, Nonlinear Anal., 70 (2009), 3307-3319. 1, 3.5 
[7] S. Y. Cho, B. A. Bin Dehaish, X.-L. Qin, Weak convergence of a splitting algorithm in Hilbert spaces, J. Appl. Anal. Comput., 7 (2017), 427-438. 1

[8] V. Colao, G. Marino, H.-K. Xu, An iterative method for finding common solutions of equilibrium and fixed point problems, J. Math. Anal. Appl., 344 (2008), 340-352. 1, 3.5

[9] P. I. Combettes, S. A. Hirstoaga, Equilibrium programming in Hilbert spaces, J. Nonlinear Convex Anal., 6 (2005), 117-136. 1

[10] S. D. Flåm, A. S. Antipin, Equilibrium programming using proximal-like algorithms, Math. Programming, 78 (1997), 29-41. 1

[11] H. Iiduka, W. Takahashi, Strong convergence theorems for nonexpansive mappings and inverse-strongly monotone mappings, Nonlinear Anal., 61 (2005), 341-350. 2

[12] C. Jaiboon, P. Kumam, A general iterative method for addressing mixed equilibrium problems and optimization problems, Nonlinear Anal., 73 (2010), 1180-1202. 1, 3.5

[13] J. S. Jung, Strong convergence of composite iterative methods for equilibrium problems and fixed point problems, Appl. Math. Comput., 213 (2009), 498-505. 1, 3.5

[14] J. S. Jung, Some results on a general iterative method for k-strictly pseudo-contractive mappings, Fixed Point Theory Appl., 2011 (2011), 11 pages. 1

[15] J. S. Jung, Iterative methods for mixed equilibrium problems and strictly pseudocontractive mappings, Fixed point Theory Appl., 2012 (2012), 19 pages. 1, 3, 3.5

[16] J. S. Jung, Weak convergence theorems for strictly pseudocontractive mappings and generalized mixed equilibrium problems, J. Appl. Math., 2012 (2012), 18 pages. 1, 3.5

[17] J.S. Jung, Weak convergence theorems for generalized mixed equilibrium problems, monotone mappings and pseudocontractive mappings, J. Korean Math. Soc., 52 (2015), 1179-1194. 1

[18] P. Katchang, T. Jitpeera, P. Kumam, Strong convergence theorems for solving generalized mixed equilibrium problems and general system of variational inequalities by the hybrid method, Nonlinear Anal. Hybrid Syst., 4 (2010), 838-852. 1, 2

[19] S.-T. Lv, Some results on a two-step iterative algorithm in Hilbert spaces, J. Nonlinear Funct. Anal., 2015 (2015), 10 pages. $1,3.5$

[20] G. J. Minty, On the generalization of a direct method of the calculus of variations, Bull. Amer. Math. Soc., 73 (1967), 315-321. 3

[21] A. Moudafi, Weak convergence theorems for nonexpansive mappings and equilibrium problems, J. Nonlinear Convex Anal., 9 (2008), 37-43. 1

[22] J.-W. Peng, J.-C. Yao, A new hybrid-extragradient method for generalized mixed equilibrium problems, fixed point problems and variational inequality problems, Taiwanese J. Math., 12 (2008), 1401-1432. 1, 1

[23] S. Plubtieng, R. Punpaeng, A general iterative method for equilibrium problems and fixed point problems in Hilbert spaces, J. Math. Anal. Appl., 336 (2007), 445-468. 1

[24] S. Plubtieng, R. Punpaeng, A new iterative method for equilibrium problems and fixed point problems of nonexpansive mappings and monotone mappings, Appl. Math. Comput., 197 (2008), 548-558. 1, 3.5

[25] Y.-F. Su, M.-J. Shang, X.-L. Qin, An iterative method of solution for equilibrium and optimization problems, Nonlinear Anal., 69 (2008), 2709-2719. 1, 3.5

[26] T. Suzuki, Strong convergence of Krasnoselskii and Mann's type sequences for one-parameter nonexpansive semigroups without Bochner integrals, J. Math. Anal. Appl., 305 (2005), 227-239. 2.6

[27] A. Tada, W. Takahashi, Weak and strong convergence theorems for a nonexpansive mapping and an equilibrium problem, J. Optim. Theory Appl., 133 (2007), 359-370. 1, 3.5

[28] S. Takahashi, W. Takahashi, Viscosity approximation methods for equilibrium problems and fixed point problems in Hilbert spaces, J. Math. Anal. Appl., 331 (2007), 506-515. 1

[29] S. Takahashi, W. Takahashi, Strong convergence theorem for a generalized equilibrium problem and a nonexpansive mapping in a Hilbert space, Nonlinear Anal., 69 (2008), 1025-1033. 1, 3.5

[30] W. Takahashi, M. Toyoda, Weak convergence theorems for nonexpansive mappings and monotone mappings, J. Optim. Theory Appl., 118 (2003), 417-428. 1

[31] J.-F. Tang, S.-S. Chang, J. Dong, Split equality fixed point problem for two quasi-asymptotically pseudocontractive mappings, J. Nonlinear Funct. Anal., 2017 (2017), 15 pages. 1

[32] S. Wang, C.-S. Hu, G.-Q. Chai, Strong convergence of a new composite iterative method for equilibrium problems and fixed point problems, Appl. Math. Comput., 215 (2010), 3891-3898. 1, 3.5

[33] U. Witthayarat, A. A. N. Abdou, Y. J. Cho, Shrinking projection methods for solving split equilibrium problems and fixed point problems for asymptotically nonexpansive mappings in Hilbert spaces, Fixed Point Theory Appl., 2015 (2015), 14 pages. 1

[34] H. K. Xu, An iterative approach to quadratic optimization, J. Optim. Theory Appl., 116 (2003), 659-678. 2.4

[35] I. Yamada, The hybrid steepest descent method for the variational inequality problem over the intersection of fixed point sets of nonexpansive mappings, Inherently parallel algorithms in feasibility and optimization and their applications, Haifa, (2000), Stud. Comput. Math., North-Holland, Amsterdam, 8 (2001), 473-504. 1, 2

[36] Y.-H. Yao, Y. J. Cho, R.-D. Chen, An iterative algorithm for solving fixed point problems, variational inequality problems and mixed equilibrium problems, Nonlinear Anal., 71 (2009), 3363-3373.1, 3.5 
[37] Y.-H. Yao, Y.-C. Liou, J.-C. Yao, Convergence theorem for equilibrium problems and fixed point problems of infinite family of nonexpansive mappings, Fixed Point Theory Appl., 2007 (2007), 12 pages. 1

[38] Y.-H. Yao, M. A. Noor, Y.-C. Liou, On iterative methods for equilibrium problems, Nonlinear Anal., 70 (2009), 497-509. 1

[39] Y.-H. Yao, M. A. Noor, S. Zainab, Y.-C. Liou, Mixed equilibrium problems and optimization problems, J. Math. Anal. Appl., 354 (2009), 319-329. 1, 1, 3.5

[40] Y.-H. Yao, Z.-S. Yao, A. A. N. Abdou, Y. J. Cho, Self-adaptive algorithms for proximal split feasibility problems and strong convergence analysis, Fixed Point Theory Appl., 2015 (2015), 13 pages. 1

[41] H. Zegeye, An iterative approximation method for a common fixed point of two pseudocontractive mappings, ISRN Math. Anal., 2011 (2011), 14 pages. 2.2, 2.3

[42] S.-S. Zhang, Generalized mixed equilibrium problem in Banach spaces, Appl. Math. Mech. (English Ed.), 30 (2009), 1105-1112. 1, 2 